\title{
Discussion on Fundamental Problems of Physics Hidden in Cosmology
}

\author{
Maurizio Michelini ${ }^{1}$ \\ ${ }^{1}$ Casaccia Research Centre, ENEA - Rome, Italy \\ Correspondence: Maurizio Michelini, Casaccia Research Centre, ENEA - Rome, Italy. E-mail: \\ m_michelini@alice.it
}

Received: July 18, 2016

Accepted: September 12, 2016

Online Published: September 30, 2016

doi:10.5539/apr.v8n5p19

URL: http://dx.doi.org/10.5539/apr.v8n5p19

\begin{abstract}
Astronomers and physicists denounce difficulties in carrying out Cosmological Research in the middle of pseudoscientific strategies. At present, Cosmological modelling follows either some revised Expanding model (i.e. Accelerating universe) or the Static-Evolving model based on the large and old universe observed in the last decades, which convinced astronomers to abandon the Big bang and the Recession of galaxies (Doppler interpretation of Hubble's redshift). Research on gravitation is made more difficult due to the presence of some misconceptions that hindered the elaboration of the Quantum theory of gravitation. Some years ago we suggested leaving the strategy of quantising Newton's pulling gravitation, since the very nature of quanta is to generate a Quantum Pushing forces between particles, due to the mutual shielding effect between two particles immersed in a uniform flux of quanta. Equating the Quantum Pushing force to the measured gravitational force, gives us the flux $\phi_{\mathrm{o}}$ and others constants of micro-quanta (see Table 1). This quantum concept in Gravitational theory allows to by pass the mathematical and physical problems that hindered the old - and not very clear - project of harmonizing Quantum Mechanics and General Relativity. Some papers published in the last decade, particularly on Applied Physics Research, showed that the flux of micro-quanta filling the universe is able to solve the following problems: 1) generation of the Quantum gravitational force (see par.6) and the Inertial forces (see par.5), 2) the homogeneous cosmological redshift (par.6.2) coming from the collisions of micro-quanta with photons, 3) the cosmic collapse - threatening the old Einstein's static universe - is prevented by the exponential attenuation theoretically required (see par.6.1) in the extended Quantum Pushing gravity, 4) all photons emitted from luminous stars are exponentially redshifted by collisions with micro-quanta and constitute the CMB, i.e. the millimetre waves uniformly coming from cosmos, measured in 1964.
\end{abstract}

These four results brought revolution in the preceding physics, as described in the Introduction. Whether the hypothesised Big bang happened or not, the enormous stellar radiation emitted in the universe must be taken into account in generating the CMB. Strangely, supporters of the Initial Explosion avoided to assess this contribution, which is anyway required because for at least 13 Giga-years all stars of receding galaxies continued their emissions A calculation (Michelini, 2013) revealed that assuming everywhere (par. 6.3) the cosmological redshift $\mathrm{z}=$ $\exp \left(x / L_{o}\right)-1$ (where $x$ is the distance of the luminous source and $L_{o}$ is the mean free path in space of micro-quanta) the energy of all redshifted photons results about equal to the measured CMB.

Part 2 shows that the same analytical form of redshift, due to the same dependence on Compton's effect, was obtained (Brynjolfsson, 2005) from the theory of plasma redshift at Large Universe. But this formulation remained useless due to the difficulty of verifying the electron plasma density at far intergalactic spaces. It was noticed that the new Cosmological redshift gives, at small distances $x<<L_{o}$, the same result $\mathrm{z} \approx x / L_{o}$ of the Hubble's redshift valid in Near Universe, demonstrating that the characteristic length $L_{o}$ does not vary across the universe. Thanks to the measurements of Planck's satellite, $L_{o}$ resulted equal to about $1.3 \times 10^{26}$. This gave credibility to Staticevolving Cosmology, where the mean free path $L_{o}$ of micro-quanta rules not only the cosmological redshift, but also the extended Quantum Pushing Gravity and the CMB cosmic background, showing the unitary structure of space.

Part 3 shows the advancement that micro-quanta Paradigm introduced in physics by correctly obtaining the relativistic equations of motion (in $\mathrm{S}$. $\mathrm{R}$ this was done on pure kinematical bases) from the dynamical balance of momentum released to particles through collisions with the flux of micro-quanta. This advancement makes free 
S.R. from the deadly paradoxes that came out along its development. A great advancement was also the establishment of the pulsating Quantum Pushing Gravity in substitution of the Newtonian Gravity.

Part 4 shows that the classical theory of the globule "collapse" proposed by Jeans in the first " 900 does not constitute a model of Star Formation. Applying that theory to the Bok's globules - discovered in 1945 - gives no definite results since the classical energy balance of the contracting globules inadequate to obtain the contraction velocity. The Jeans' hypothesis of the free fall contraction appears ridiculous. The observational evidence that Bok's globules are incubators of stars needs an adequate theoretical model of Star Forming which allows to calculate the Incubation time. The gravitational accretion of galactic gas upon an extinct star, was developed to explain the formation of obscure Supermasses in AGN's. The calculated incubation times resulted well higher than the Big bang age of universe. Accretion can be also adopted for small inert masses (fragments of Supernovae, cosmic powder, planetary nebulae, etc) giving rise to Star Forming models (par. 9 bis).

In Part 5 is pointed out that any kind of quanta colliding with particles release some energy that is ruled by Compton's equation. Calculating the energy that micro-quanta of pulsating Quantum Pushing Gravity due to the Hydrogen nucleus, release at regular intervals on the electron, it is found an average power $p_{e} \approx 2 \times 10^{-54}$ watt. This is not a Hypothesis, but a result standing on well founded Principles of Physics.

Keywords: Special and General Relativity, Micro-quanta and Quantum Pushing gravity, Non-Doppler Hubble's redshift, Cosmological redshift in Far universe, Star Formation models, Incubation time of stars.

\section{Introduction}

After the advent of General Relativity, the first models of Universe represented a static universe. Einstein itself elaborated in 1917 a relativistic static model, but he recognised that such universe would have collapsed under the gravitational force. So he assumed empirically that some unknown repulsive force (referred as the so called Cosmological constant) were present on the large scale, to avoid the cosmic collapse of the Static model. Some expanding models were developed in 1924 by the Russian mathematician Alexander Friedman, but the physicists considered them as curiosities. The publication in 1929 of the Hubble's measurements of redshifts of galaxies leaded to the intuitive Doppler interpretation based on the recession of galaxies and convinced Einstein to throw the Cosmological constant in the waste basket. Significantly, Hubble did never take sides on the dispute. Strictly speaking, the redshifts he measured was increasing with the distance of galaxies. This was correct, but not exciting. Conversely, the increasing recession velocity of galaxies (Doppler) became most popular. Following the archetype of the explosion of a "primordial Egg" advanced in 1927 by G. Lemaitre, (a catholic priest, Harvard laureate) many relativistic models were elaborated in a decade. Let's recall, for instance, the Einstein-De Sitter Expansion model, which retained the Cosmological constant. Why? These models were characterised by the fact that galaxies may repeat the cycle expansion-contraction under the opposite forces of inertia and gravitation. During the contraction phase, the presence of the Cosmological constant appeared necessary to avoid the cosmic collapse.

The subsequent Big bang model accepted even the old Lemaitre's archetype, but it appeared more affordable thanks to the theory of stellar nucleosynthesis, elaborated in the meantime by Alpher and Bethe. However the idea of the Cosmic Egg, which exploded some time ago, did not convince. The related Cosmology cancelled a century of preceding observations on the ages of various astronomical systems (globular star clusters, galaxies, clusters of galaxies). Many alternative cosmologies arised to restore the flux of ideas coming from astronomy.

At present, the Static-evolving-large universe did reject the Doppler interpretation of the cosmological redshift, recognised as the greatest trap in the history of Astronomy. Some attempts were proposed without success (tired light) to explain the cosmological redshift. However the new revolution of micro-quanta cannot be easily refused, because it reinforce current physics as well as the Copernican revolution enhanced the physics of Renaissance (entrapped in the Geocentric culture) opening the door to the physics of Galileo and Newton.

Part 3 shows that the advancement in physics introduced by the Micro-quanta consents to obtain from dynamical principles the relativistic equations used for studying eminently dynamic phenomena (e.g. the increase of mass) arising from collisions with micro-quanta to the motion of particles (see par. 7). Let's recall that the kinematical basis gave Special relativity a weak structure that was unable to resist several ad hoc paradoxes denouncing some absurd results. The new formulation of Relativity restores the natural frame of space and time where happen all physical phenomena whose laws are correctly known. In particular the application of Special relativity to the Gravitational phenomena showed a particular metrics that distorted space suggesting it was "curve", whereas the gravitational properties of space are dependent only on the micro-quanta.

The micro-quanta Paradigm recognised the right role of the Gravitational force abolishing the "gravitational mass" and changing the Newton's pulling interaction in the pulsating Quantum Pushing force, so introducing Gravitation 
between the Four Fundamental forces of nature supported by proper interaction waves. The explanation of the Inertial forces from physical collisions between particles and micro-quanta gives to the masterly Newton's Law of inertia the character of a fully accurate equation (even to the sub-nuclear scale) which is calibrated to explains (par. 7.1) the presence in space of micro-quanta. Newton ignored this (unknown) reality, but his masterly Law of Inertia incorporates their properties (par.7.1).

In the last years the scientific community suffered troubles due to ovations bestowed by the mass-media to some so called great Discoveries, while the opinions of skilled scientists remained sceptical.

Recently Otto Rossler published "A Trap posed by Nature to Humankind" recalling that after the Special relativity advent, nature put a trap to the physical science (Rossler, 2016). Five implications of Einstein's theory overlooked the research, changing our self-understanding of physics. This author also commented as a "Fraudolent Joke" the so called Discovery of Gravitational Waves from Cosmos in a letter to New York Times in February 18, 2016. Worth of attention is the work by S. Crothers "A critical analysis of LIGO's recent detection of GW caused by merging Black holes" which points out the questionable theoretical assumptions upon which the research is based (Crothers, 2016).

In Part 4 the research of a correct model of gravitational accretion is carried out (par.9) through a calculation based on Boltzmann's Transport theory in gases, showing star incubation times going from some Giga-years up to hundreds of Giga-years when the initial inert mass is too small. An application of these result to the Milky way, which shows in last decade an average rate of 10-11 Novae per year registered by the Intern. Astronomic Union (CBAT, Harvard 2010), demonstrated that the time elapsed from the first star appearance up today equals 38.1 Giga-years. This puts questions on the so called age of universe. A wide research on star forming regions is currently developed at Alma telescope (Chile), as reported in MNRAS Oxford Journals (MNRAS, 2016).

Part 5 calculates the physical power released on the electron of Hydrogen atom through the pulsating Quantum Pushing Gravity. Enlarging the calculation (par. 10) to all nucleons within the first flight path $l_{1}=1 / \mathrm{A}_{0} \delta$ comprised in the surrounding spherical mass $\mathrm{M}_{1}=4.18 \delta l_{1}^{3}$, we obtained at first approximation that each electron receives the power $p_{e} \approx 1.23 \times 10^{-46}\left(\mathrm{M}_{1} / l_{1}^{2}\right)=1.23 \times 10^{-46}\left(4.18 \delta l_{1}\right)$ watt which, substituting, finally gives $p_{e} \approx 1.23 \times 10^{-}$ ${ }^{46}\left(4.18 / \mathrm{A}_{\mathrm{o}}\right) \approx 1.23 \times 10^{-35}$ watt. It is expected that the energy accumulated on the electrons generates photons through the Hydrogen IR emission lines, particularly the series of Balmer, Paschen, Brakett, Pfund. This result gives a scientific relevance to the observations of Luminous Infrared Galaxies (LIRG, emitting more than $50 \%$ on IR frequencies) since they are more numerous than Starburst galaxies.

\section{Part 1. Near-universe Cosmology Based on the Hubble's Redshift}

\section{The Big Bang Model}

This model, which took its name from a colloquial expression of the astronomer Fred Hoyle, became popular in the ' 50 through the books of G. Gamow, an appreciate author of science-fiction.

Up to date the expansion of universe is not yet experimentally observed because this requires measuring two times the distance of any far galaxy after an adequate interval of time (by necessity of the order of the human lifetime). An attempt would presently give two distances whose difference is negligible comparing with the experimental errors. Strictly speaking, we don't know either the galaxies are receding, or are approaching. As a consequence, all models of expanding universe have to assume some physical phenomenon that links the observed Hubble's redshift to the recession of galaxies. The most simple and popular link is obviously the Doppler effect, a phenomenon well verified on the Earth. However when we inquire on the distances involved in cosmological redshift, we have to admit that several physical phenomena may reduce the photon energy (see : tired light) considering the great time duration of the photon travel. Besides, since the cosmological redshift always increases with the source distance, we have to consider phenomena that act along all photon travel. This requires to introduce something better than the obsolete Cosmological constant. We are convinced that an adequate solution would be to introduce the physical Interaction due to the micro-quanta flux that works uniformly in the universe, giving everywhere rise to the Inertia of bodies and to the Quantum Pushing gravitational force.

The scientific content of Big bang was impressive at beginning: the high-energy radiative explosion occurred within $10^{-35}$ seconds, while all Hydrogen and Helium of the universe were generated within 3 minutes. So scans the mythology of the Big bang. Mythology wants to abandon the rational reasoning. The model was ennobled in 1965 after the discovery of the CMB, that R. Dicke interpreted as the relict of the primordial explosion. However the whole stellar radiation that was emitted within $13.7 \mathrm{Gyr}$ is not taken into account to explain the measured CMB.

The weak side of this model remains the short age that Big bang assigned to the universe, opening serious problems to all astronomers whose works on the globular star clusters and the evolution of Milky Way showed an age 
considerably higher. A model to calculate the age of our galaxy starting from new measurements of the "obscure" mass hidden in the Milky Way, may be devised by using the secular statistical data on the observed generation of Novae in our galaxy (see par. 9 bis). The last published data by the Intern. Astronomic Union (CBAT Harvard, 2010) show about 10-11 Novae per year, due to the rapid increase of observed data from the recent installation on satellites of wide-spectrum telescopes. Being the last data fairly higher than the rate observed in the ' 60 , it is reasonable to assume the above figure as the most accurate as today possible.

During last decades of XX century, the Big bang model received theoretical improvements to reinforce the "initial explosion" that gave rise to great problems. This anomaly was treated in the ' 90 by the Inflation theory, which made recourse to hypothetical radiation velocities much higher than the speed of light, so appearing a pure mathematical support to the Big bang model.

Astronomers are convinced that the flux of empirical knowledge (in the past directed from Astronomy towards Cosmology) suffered after the BB cosmology a re-direction which generated some counter-blows in the astronomic research. The recent astronomic observations revealing a very large universe, such as the super clusters of galaxies discovered by B. Tully and J. Fischer, required much time to be realised through gravitation. Other recent observations showed that the distribution of galaxies tends to alternate crowded regions to large spaces practically void (Baryon Acoustic Waves). Other interesting objects are the Supermassive Obscure bodies observed within the AGN of some galaxies. In our Milky Way there is an obscure Supermassive body (Schoedel R. Ott T. et al., 2002) equal to $3.7 \times 10^{6}$ solar masses, so the physicists debates if this huge mass was really accumulated by gravitational accretion within the short BB age. The last generation of astrophysicists accumulated a lot of new data from the enlargement of the observations due to orbiting telescopes operating in the infrared as well as in the visible and $\mathrm{X}$ rays. The remarks to maintain open the door to new coming observations appears right and wise (Lopez Corredoira, 2003).

The Luminous Infrared Galaxies (LIRG) emit more than $50 \%$ of energy on IR frequencies and are more numerous than starburst galaxies. A question arises: If the IR emission is prevalent in LIRG that are prevalent among galaxies, then the total radiation generated directly from IR sources is greater than the total stellar radiation. Are there autonomous sources of IR radiation?

\subsection{The So Called "Expansion of space" and Other Misconceptions}

In recent years some physicists extended the superluminal velocities of radiation even to the mass of the receding galaxies. This sparkling idea gave rise to negative comments between the physicists which didn't accept such a by-pass of the relativistic laws of inertia. The velocity of light does not depend on the velocity of the galactic source. So, whatever the type of repulsive force acting between galaxies, this force travels with the velocity $c$ of the interacting quanta (action at-a-distance is banished) and ceases when the galaxies tend to the velocity c. This is why the expansion of galaxies cannot be accelerated over c. The physicist Steven Weinberg and the cosmologists Martin Rees gave their comments on the New Scientist (1993) about the "expansion of space" which leads to superluminal receding velocities. "How is it possible for space, which is utterly empty, to expand?" asked Weinberg. Supporters of the Expansion of space (conscious that Special relativity does not accept superluminal velocities) make recourse to General Relativity which, on their words, accepts the expansion of space. However the recourse to G.R. involves higher questions, because it was conceived as a theory of the astronomical observations, which require specific metrics based on the role of E.M. signals in transferring the astronomical phenomena up to our eyes, as clearly demonstrated by the "gravitational lens".

As we shall see, superluminal velocity is not unique among the misconceptions that poisoned physics during past centuries. Newton was an isolated pioneer which, although not knowing Quantum theory, was able to develop a complete theory of the gravitational phenomena a century before the measurement of the gravitational constant G. Some misconceptions was inevitable in dealing with the concepts accepted in his times. This is the case of the gravitational mass conceived as "source" of gravitation. Any Quantum-gravitational force do not pull the masses each other, because quanta (by their very nature) push the masses each other (par. 6.1). So the old "Pulling" gravity conceived at Newton's time, is presently a misconception that generates unphysical entities, such as the so called black holes. To day Newton's gravity has been substituted by the pulsating Quantum Pushing gravity (par.6).

The improper use of the (obsolete) Cosmological constant is equivalent to a misconception. In fact the extended Quantum Pushing gravity shows at cosmological distances an exponential attenuation $\exp \left(-x / L_{0}\right)$ reducing the gravitational force. This means: no hypothetical repulsive forces (the old argument by Einstein) but real attenuation of the gravitational force at cosmological distances due to mutual collisions of micro-quanta, whose mean free path is $L_{0}$. 
As pointed out in the paper "Six Baseless and Irrational Problems in Modern Cosmology" (Li, 2015), the cosmologists are still using some baseless concepts such as: Cosmic explosion, Recession of galaxies, Arbitrary multidimensional space, Superluminal velocities, Curved space, Black holes. Concluding his review, this author sustains it is necessary to regain the Newtonian concepts of time and space, to revise the present physics.

\subsection{The Accelerating Universe and the Problem of "Dark" Matter}

It would represent a change on cosmology, but is largely based on some unphysical concepts. The Nobel prize 2011 for Physics was awarded three astronomers which claimed the "discovery" of the accelerated recession of galaxies through the calculation - by means of an expanding model Lambda-CDM - of the present distances of far Supernovae 1a, whose redshift they had previously observed through a specific study of their luminosity evolution. That procedure consents to calculate how many years ago the luminous explosion did occur because their distances may be computed because light travels with velocity c across non expanding universe. Introducing this procedure in Lambda-CDM model based on the Expansion of Space (with superluminal velocities) means that it is impossible to know if the galaxies are really accelerating or not. In the work presented by the three astronomers there are many high-sounding concepts, but no real knowledge that authorises to speak of discovery. Confusion arises from the concepts of "obscure" energy (which is not defined) and "dark" matter used in the sense of an unknown kind of matter.

This "dark" matter differs from the plain concept coined by Zwicky in 1930 measuring the velocity of the speedy stars of galactic halo, which denoted a low-luminosity mass amounting to 5-6 times the luminous mass, due to the extinct luminous stars of Milky Way. To day this old discovery has been entirely confirmed by new astronomical observations (Gnedin et al., 2010) that measured the excess velocities of the speedy stars in the range $25<r<80$ $\mathrm{kpc}$. The circular velocity profile declines very little, less than $20 \%$ over an order of magnitude in radius. Such a profile requires a massive and extended presence of not visible stars that increase the total mass to $6.9(+3.0-$ 1.2) $\times 10^{11}$ solar masses.

Why the so called Dark Matter particles are present in the galactic region where in remote past were present billions of luminous stars? There are many types of obscure stars, i.e. old stars that lost their luminosity, but not their mass. After leaving the Main sequence, all ancient dense stars (i.e. dwarf stars of different colour index described in the Hertzsprung - Russell diagram) show a continuous decrease of luminosity. The "obscure" mass in Milky Way results to day, according to the measurements of the Planck satellite, about 5.66 times greater than the mass of the luminous stars. This agrees well with the ratio found by Zwicky in 1930 . Obviously this great obscure mass implies a galactic age, fulfilling the presently observed rate of Novae generated in Milky Way (10 $\div 11$ per year), much greater than the age predicted by Big bang. It is confusing to depict the astronomical discovery of the low-luminous "extinct" stars as a kind of unknown matter not yet observed. The problems of Physics are so numerous that deviating the interest of young researchers towards some myths is a dissipating practice. A well deserving onlineEncyclopaedia describes in this way the still unsolved problems in physics:

- Cosmology and General relativity

- Quantum gravity

- Particle physics

- Astronomy and astrophysics

- Physics of the fundamental forces

- Biophysics

\subsection{CMB: What happened to Stellar Emissions in the Expanding Universe?}

After the discovery of CMB in 1964, the Big bang cosmologists explained this background radiation (i.e. EM waves coming from all sky vault) as the thermal radiation left over from the time of recombination of particles in neutral Hydrogen. However they forgot to explain what is the sort of the total stellar radiation emitted in the universe. In the following decades it became clear that CMB waves, showing a perfect blackbody spectrum, originate also from the redshifted photons emitted from stars, whose light shows notoriously a blackbody spectrum. Richard Tolman showed in 1934 that thermal radiation may cool in space without losing the blackbody spectrum. This means that within the measured $\mathrm{CMB}\left(2.72^{\circ} \mathrm{K}\right.$ peak $)$ are present even the redshifted waves coming from stellar photons. A recent calculation showed (M. Michelini, 2013) that applying the cosmological redshift of static universe (energy reducing factor $1+\mathrm{z}=\exp \left(x / L_{o}\right)$ to photons coming from all universe (even beyond the time frontier of $13.7 \mathrm{Gyr}$ ) produces in whatever point of space a flux of millimetric E.M waves whose energy is about the same of CMB. The reduced photon energy depends really from the exponential cosmological redshift due to 
the collisions of micro-quanta. On the other hand, the Big bang model neglected the stellar radiation affirming that all CMB is the relict of the cosmic explosion, but this cannot proved because the link with the cosmic explosion is not known. Some BB cosmologists sustain that Expanding universe is supported by the Olbers' paradox, which requires that cosmologic models must guarantee the effective darkness of the night sky. To this subject they recall that Doppler effect reduces the energy of photons emitted (even in the night) from receding galaxies. However the relativistic Doppler effect appears to reduce the galaxies radiation of about $31 \%$ only. This is not sufficient to reach the darkness of the night sky.

\subsection{Micro-Quanta Reduce Really the Photon Energy}

It is time of making clearness on the CMB coming from stellar radiation. The Cosmological redshift is a mechanism due to the flux of micro-quanta (see par. 6.2) that really reduces photon energies through collisions, gradually disintegrating their weak structure. So the energy content of photons returns to the Great Cycle of energy between matter and free micro-quanta, which in turn release energy - via Compton's effect - in collisions with particles, especially electrons, as recalled in Part 5 of this paper.

In Large static-evolving universe, the energy of coming stellar photons is redshifted exponentially as shown in par.6.3. Contrary to the $\mathrm{BB}$ calculation of the $\mathrm{CMB}$ produced by the redshift of photon emitted from receding galaxies, the exponential attenuation really allows to reach the night sky darkness required by the Olbers' paradox.

The redshift suffered by the stellar photons producing the $\mathrm{CMB}$ peak $\left(2.73^{\circ} \mathrm{K}\right.$ ) equals about $\mathrm{z} \approx 2000$ (see par. 4). In the same paragraph Equation (4) gives the distances of Galaxies $x=L_{o} \ln (1+2000)$ that can be observed through the redshift of the associated Supernovae in a quasi-static universe. So one finds that CMB waves did travel (eq.4.6) a distance equivalent to 104 Giga light-years. For the first time CMB waves allow to calculate a definite size of the stellar universe.

\section{Steady state models}

Appearing little later the Big bang. this model was considered more reliable since it refuses the embarrassing initial explosion. In fact it assumes a very old universe, where the stationary expansion - linked to the Hubble's redshift - is forced by the gradual creation of matter in new galaxies, while some mechanism maintains the average distances between galaxies and makes constant the average density of the universe. Subsequently this model has changed in Quasi Steady state (Narlikar, 1990) where the creation of matter has been substituted by an increment of thermal energy of the galactic gas throughout the universe. Although interpreting the Hubble's redshift as a proof of the expansion of universe, this type of models consider a very large and ancient universe that appears more suitable to lead towards the static evolving universe,

\section{Part 2. Large-Universe Based on High Redshifts Observed Through Supernovae 1a}

\section{The Theory of Plasma Redshift}

The unphysical features of the Big bang make necessary a revision of the Doppler interpretation of Hubble's redshift, the greatest trap that Nature put on the path of Science.

The redshift observed using far Supernovae $1 a$ as "standard candles" placed within far galaxies, gave rise to new cosmological models (Brynjolfsson, 2005) describing a very old, very large, quasi-static universe. In his work on plasma physics, Brynjolfsson wrote: "Plasma redshift explains the solar redshift, the galactic corona, the cosmological redshift, the CMB radiation". In a next paper he verified (Brynjolfsson A, 2006) that the redshift of the observed SNe embedded in far galaxies increase exponentially along the distance $x$ travelled by photons across the intergalactic plasma

$$
1+\mathrm{z}=\exp \left(x \mathrm{~N}_{\mathrm{e}} / 3 \times 10^{28}\right) .
$$

From this relation (where $\mathrm{N}_{\mathrm{e}}$ is the average electron density in the intergalactic plasma) the distance $\mathrm{x}$ can be obtained in function of the redshift

$$
x=\left(3 \times 10^{28} / \mathrm{N}_{\mathrm{e}}\right) \quad \ln (1+\mathrm{z}) .
$$

This algorithm extends correctly the Hubble's law to the large universe. Obviously the small distance $x \approx z\left(\mathrm{c} / \mathrm{H}_{0}\right)$ defined by the Hubble's law must be equal to the distance $x=z\left(3 \times 10^{28} / \mathrm{N}_{\mathrm{e}}\right)$ given by the general Equation (4.2) when $z<<1$, that is

$$
L_{o}=\left(\mathrm{c} / \mathrm{H}_{\mathrm{o}}\right)=\left(3 \times 10^{28} / \mathrm{N}_{\mathrm{e}}\right)
$$


This equality allowed Brynjolfsson to solve the problem of determining the average electron density in the intergalactic plasma. This solution puts obviously some doubts on the real dependence of redshift on the intergalactic plasma, which is irregularly distributed in association with the active regions of universe. Substituting in Equation (4.3) the Hubble's length $L{ }_{o} \approx 1.31 \times 10^{26}$ measured by Planck's satellite, one obtains the explicit distance of a galaxy with known redshift

$$
x=L_{\mathrm{o}} \ln (1+\mathrm{z}) \approx 1.31 \times 10^{26} \ln (1+\mathrm{z}) .
$$

This makes clear that redshift depends uniformly on the spatial distance, that is depends on a uniform physical reality.

Analogously, the redshift for a source of known distance is given by

$$
1+\mathrm{z}=\exp \left(x / L_{\mathrm{o}}\right)
$$

that throws new light on the Hubble's length $\left(L_{o}\right)$ which characterises the intergalactic medium, opening the door to the flux of micro-quanta filling the universe (par.5). This one guarantees both the cosmological redshift and the presence of the physical inertial forces acting on atoms, nuclei, stars, etc.

No doubts that eqs(4.4 - 4.5) individuate a Static-evolving universe whose results agree with several astronomical observations that the standard Big bang model does not predict, especially on the age of all astronomical objects.

Assuming that photons coming from far stars suffer scattering from the intergalactic medium, an overall redshift $z \sim 2000$ (typical value for CMB originated from stellar photons, assuming an average star temperature of $5500^{\circ} \mathrm{K}$ to be compared with the $\mathrm{CMB}$ observed temperature $2.73^{*} \mathrm{~K}$ ) must be taken into account. Then, one may calculate with Equation (4) the time elapsed from the photon emission up to present

$$
\Delta \mathrm{t}=\mathrm{x} / \mathrm{c}=\left(1.31 \times 10^{26} / 3 \times 10^{8}\right) \ln (2001)=3.32 \times 10^{18} \mathrm{sec} \sim 104 \mathrm{Gyr} .
$$

This is the time taken by the CMB waves travelling from ancient stars up to present. It represents also a minimum measure of the size of quasi-static universe, in the sense that all more distant galaxies do not contribute sensibly to the CMB energy $\left(2.73^{\circ} \mathrm{K}\right)$ measured on the Earth.

\subsection{Which is the Medium Filling the Intergalactic Space?}

The correct result introduced by Equation (3) and subsequently by Equation (5) is worth of profound reflection. The theory of Plasma redshift affirms that the interaction between photons and the intergalactic medium follows the Compton's scattering. This put the following question: Which is the new significance of the term $L_{\mathrm{o}}$ (Hubble's length) which does no longer introduce any alleged velocity of the galaxies?

We don't know the real electron-density in different intergalactic spaces. On the other hand, we know that Equation (1) shows the regularity of the redshifts observed across the sky vault at any given distance of the source, disregarding the type of collisions suffered by photons. This consents to assume an uniform intergalactic medium that generates the observed redshifts, as stated in Equation (5). The candidate medium has been recognised (The new cosmology, 2013) to be the flux of micro-quanta that fills uniformly all space, determining Gravitation and Inertia in the universe.

We know that Compton's scattering rules also the micro-quanta colliding with two mutual-shielded particles immersed in the flux, so giving rise to the Pushing gravity force (see par. 7.1). Obviously some effects rise from the collisions between the flux of micro-quanta and single E.M. quanta. What happens when micro-quanta collide continuously on a travelling photon? An electromagnetic photon (weak structure scattered and absorbed by matter) is likely constituted by an assembly of polarised micro-quanta weakly linked among them. In this case the free micro-quanta (i.e. the flux $\phi_{0}$ ) collide with the weakly-linked quanta constituting the photons. Having about the same energy, one free micro-quantum scatters away (on thermodynamic average) one polarised micro-quantum, so reducing the photon energy. This is the reason why the collisions with the uniform intergalactic medium give rise to the cosmological redshift depending exponentially on the distance of the source (see par. 7.2). Since the Hubble's length is the m.f.p. of micro-quanta, this makes free Cosmology from the time-dependent Hubble's "constant" $\mathrm{H}(\mathrm{t})$ that puzzled the astronomers in expressing the relation between the redshift and the alleged receding velocity of galaxies.

\section{The Physical Generation of the Inertia of Bodies}

Obviously, particles constituting the intergalactic medium undergo the same forces experienced in the laboratories. Considering that the wavelength of the micro-quanta is much smaller than the size of particles (represented by 
their small cross section (to micro-quanta) amounting in the case of protons to $\sigma=8.57 \times 10^{-38}$ ), the flux of microquanta can be also the source of the Inertia of particles. This solves the long standing problem of the local source of the inertia of bodies. Let's recall that in the past century some of the opponents believed, on the contrary, that the (instantaneous) inertial forces might come from the far masses of the universe (Mach).

At present the problem of the Inertia of accelerating/decelerating particles is considered on local bases, since great inertial forces arise instantaneously in the collisions between high-energy particles. Special Relativity is put in doubt because the physical genesis of these inertial forces is not yet clear.

We require that small quanta must have small energy and very small wavelength to make possible the generation of the Inertia of particles through a great number of isotropic simultaneous collisions. We named "micro-quanta" the waves whose wavelength equals the Planck's length $\lambda_{0}=4.05 \times 10^{-35}$, a value that assures the Inertia of particles through a great stable number of micro-quanta in simultaneous collisions.

This is the reason why we support the local genesis of the inertial forces depending on the relative velocity $\mathbf{v}$ between particles and the local density of micro-quanta. To make an intuitive comparison, let's consider a massive body moving across the low density atmosphere. Of course the comparison is not correct, because the mean free path of air molecules is very short (around $10^{-4}$ ), so all molecules rapidly originate an increasing pressure on the body. On the contrary the mean free path of micro-quanta is extremely long (about $10^{26}$ ) so each of them contrasts the motion of body singularly. This comparison shows the necessity of defining just the relative velocity of a particle towards the surrounding cloud of micro-quanta.

\subsection{The Local Generation of the Inertial Forces}

The most crucial proof of the local generation of inertial forces (for instance the collision between high-energy particles) is to verify the amount of energy that the inertial force, expressed by the Newton's masterly equation $\boldsymbol{F}=\mathrm{m}_{\mathrm{o}} \mathrm{d} \mathbf{v} / \mathrm{d} t$, requires to brake the particles during collisions. The opponents of the local genesis of inertial forces affirmed the impossibility that a small region of space might contain the amount of energy necessary to sustain the inertial forces. Let's consider the violent collision between protons accelerated in LHC to the energy $\mathrm{E}_{\mathrm{N}}=50 \mathrm{Gev}$ $=8 \times 10^{-9}$ and nucleons of the crust. Experiments of non charged particles produced at LHC and sent to the National Laboratories of Gran Sasso (GSNL) have been performed in last years to study the properties of high-energy neutrinos in deep path $(720 \mathrm{~km})$ trough Earth crust. Neutrinos are waves with a non-zero rest mass, which have been detected at low energy from the Supernova of 1987 and usually from the Sun, where their number is about $30 \%$ less than expected from the emission calculated for the p-p nuclear fusion. Let's assume that Neutron is constituted by a. pair Proton-electron tightly bound under the Strong force. In the collision with crustal Neutrons, Proton strips the electron, and continues traversing the crust at velocity near that of light as a neutral particle, so someone spoke of $50 \mathrm{Gev}$ Neutrinos. However measurements at GSNL showed that during path under the crust, these "neutrinos" reduce their energy from 50 up to $17 \mathrm{Gev}$ ! We observe that this relenting appears to agree with that of the fast neutrons $(14 \mathrm{Mev})$ emitted by fission of uranium. This is obviously a simple comment that tends to reduce the excessive number of properties attributed to neutrinos.

Since neutral particles have no charge, the collision with matter is ruled by Strong and Inertial forces. The pressure of micro-quanta incident on the nucleon cross section (about $10^{65} / \mathrm{sec}$ ) imposes a minimum distance between the particles. From the definition $\mathrm{A}_{\mathrm{o}}=$, it is easy to show that assuming for particles the Compton's wavelength $\lambda_{\mathrm{i}}=$ $\mathrm{h} / 2 \pi c m_{i}$, the product $\left(\sigma_{\mathrm{i}} \lambda_{\mathrm{i}}\right)$ equals for any particle the volume

$$
\mathrm{V}_{\mathrm{o}}=\left(\sigma_{\mathrm{l}} \lambda_{\mathrm{i}}\right)=\mathrm{hA}_{\mathrm{o}} / 2 \pi c=1.81 \times 10^{-53}
$$

which might be considered the portion of space that generates the inertial forces of each particle. The problem is still debated. The micro-quanta paradigm states that the energy density $\mathrm{p}_{\mathrm{o}}=\mathrm{E}_{0} \phi_{0} / \mathrm{c}$ of micro-quanta in space equals $4,33 \times 10^{61} \mathrm{Joule} / \mathrm{m}^{3}$. We have to find the cross section of the particle that is linked to $\mathrm{p}_{\mathrm{o}}$, which represent also the radiation pressure exerted by micro-quanta on particles. The incredibly high energy density $p_{o}$ is made possible tanks to the fact that micro-quanta, showing a mean free path equal to the Hubble's length $\left(1.3 \times 10^{26}\right.$ metres $), d o$ not practically collide each other within the small unit of volume $\left(1 \mathrm{~m}^{3}\right)$. On the contrary, micro-quanta collide independently on particles since their charge is dominant.

The Principle of inertia for a mass travelling with uniform velocity becomes now clear, since the moving mass receives from the incident flux, due to its isotropy , the same number of collisions both from left and from right of its path. In absence of other external forces, any moving mass does not deviate from a strait line. In par. 7.1 it will be shown the tight accord between the Newton's Law of Inertia with the present local generation of inertial forces. 


\section{5.bis. Some Elements about the Inertial Model of Particles.}

The mass of a stable particle is due to collision with micro-quanta scattered by the electric charge at distance $r_{\mathrm{o}}$ from the charge, so the spherical surface $4 \pi r_{0}{ }^{2}=4 \pi \sigma$ is defined as cross section respect to the isotropic flux of micro-quanta. Since the energy $E_{o}$ is extremely lower than the mass-energy of the particle, Compton's scattering establishes that a micro-quantum if optically reflected by particles. The momentum released on the particle by a micro-quantum diffused with angle $\alpha$ has modulus $|\Delta \mathbf{q}|=2\left(\mathrm{E}_{\mathrm{o}} / \mathrm{c}\right) \sin (\alpha / 2)$ and is radially inward directed.

There is a statistical (nearly perfect) equilibrium between two momenta released upon two points diametrically opposite. This condition is widely satisfied considering the high rate of incident micro-quanta (about $\approx 10^{65} / \mathrm{sec}$. upon a nucleon). The interaction of particles with the flux of micro-quanta introduces the concept of simultaneous collision with $\mathrm{N}_{\mathrm{i}}=\sigma_{\mathrm{i}} \phi_{\mathrm{o}} \tau_{\mathrm{o}}$ micro-quanta, where $\tau_{0}=\lambda_{0} / \mathrm{c}$ is the average time spent by each scattered microquantum in changing its direction, so that a stable particle may be defined as a stationery state of interaction with micro-quanta flux. The micro-quanta wavelength $\lambda_{0}$ is calculated in Table 1 after knowing the second Planck constant $h_{0}$. Then $N_{i}$ equals about $10^{22}$ in the case of nucleons, justifying the nearly perfect equilibrium in the interaction. The mass of a particle is defined coherently with the Newtonian definition of mass. The micro-quanta scattered at each point of the surface determines on the average the fraction of the particle mass along a generic radial direction

$$
\Delta m_{\mathrm{i}}=\Delta \mathrm{q} / \Delta \mathrm{v}=2\left(\mathrm{E}_{\mathrm{o}} / \mathrm{c}\right) \sin (\alpha / 2) / 2 \mathrm{c} \sin (\alpha / 2)=\mathrm{E}_{\mathrm{o}} / \mathrm{c}^{2} .
$$

Since the number of scattered micro-quanta in a simultaneous collision is $\mathrm{N}_{\mathrm{i}}$, the particle mass results

$$
m_{\mathrm{i}}=\mathrm{N}_{\mathrm{i}} \Delta m_{\mathrm{i}}=\sigma_{\mathrm{i}} \phi_{\mathrm{o}} \tau_{\mathrm{o}}\left(\mathrm{E}_{\mathrm{o}} / \mathrm{c}^{2}\right)=\sigma_{\mathrm{i}} \mathrm{p}_{\mathrm{o}} \tau_{\mathrm{o}} / \mathrm{c} .
$$

This gives the fundamental ratio $\mathrm{A}_{\mathrm{o}}=\sigma_{\mathrm{i}} / m_{\mathrm{i}}=\mathrm{c} / \mathrm{p}_{\mathrm{o}} \tau_{\mathrm{o}}=\mathrm{c}^{2} / \mathrm{p}_{\mathrm{o}} \lambda_{\mathrm{o}}$ valid for any particle. Writing the particle mass

$$
m_{\mathrm{i}}=\mathrm{N}_{\mathrm{i}}\left(\mathrm{E}_{\mathrm{o}} / \mathrm{c}^{2}\right)
$$

suggests a simple interpretation of the electromagnetic energy of particles

$$
m_{\mathrm{i}} \mathrm{c}^{2}=\mathrm{N}_{\mathrm{i}} \mathrm{E}_{\mathrm{o}}
$$

that equals the energy of the $\mathrm{N}_{\mathrm{i}}=\sigma_{\mathrm{i}} \phi_{\mathrm{o}} \tau_{\mathrm{o}}$ micro-quanta scattered in simultaneous collisions. Then any stable particle can be defined as the stable state of the interaction with micro-quanta. This appears to give a clear answer to the problem of the mass origin (Higgs mechanism). This will be examined in a next paper.

\section{Genesis of Newton's Gravitational Force through the Flux of Micro-Quanta}

The micro-quanta Paradigm is not a tool devised to analyse only the influence of Quantum Gravity on Cosmology. It is above all a paradigm elaborated to introduce new solutions to some classical problems through the Quantum Gravitational theory and Quantum Inertial forces. In general a high number of micro-quanta hit the particles (for instance a nucleon receives $\sigma_{\mathrm{n}} \phi_{\mathrm{o}} \approx 6 \times 10^{65}$ collisions/sec) but only a little fraction of non-balanced micro-quanta transmit momentum and energy to the particles, since in general the effects of any incident micro-quantum are cancelled by an other symmetric micro-quantum with opposite direction.

In 2010 it was shown (Michelini, Nov. 2010) that two adjacent particles immersed within a isotropic flux of ordinary quanta of light (and is valid also for micro-quanta) undergo the mutual shielding-effect - since microquanta are scattered by particles - that generates a force pushing each other the particles. The paper defines the Quantum Pushing gravity

$$
F_{g r}=\left(2 \mathrm{E}_{\mathrm{o}} / \mathrm{c}\right) \Delta \sigma \phi_{0}\left(\Delta \sigma / 2 \pi r^{2}\right)
$$

where $\left(2 \mathrm{E}_{0} / \mathrm{c}\right)$ is the momentum leaved by each recoiling micro-quantum. This pulsating force is generates by the flux $\phi_{o}$ of micro-quanta, whose value is chosen in order to reproduce the measured Gravitational force between two nucleons (constituting $99.8 \%$ of the neutral mass) which can be put in the canonical form introducing the uniform radiation pressure $\mathrm{p}_{\mathrm{o}}=\mathrm{E}_{\mathrm{o}} \phi_{\mathrm{o}} / \mathrm{c}$ of micro-quanta

$$
F_{g r}=\sigma_{\mathrm{n}} \mathrm{p}_{\mathrm{o}} \mathrm{K}_{\mathrm{n}}\left(\sigma_{\mathrm{n}} / 2 \pi r^{2}\right)=G m_{n}{ }^{2} / r^{2}
$$

where $\mathrm{K}_{\mathrm{n}}=\left(\Delta \sigma / \sigma_{\mathrm{n}}\right)^{2}=1.83 \times 10^{-51}$, since $\Delta \sigma=\mathrm{x}_{0}^{2}=3.67 \times 10^{-63}$ as shown by eq $(10.3 b i s)$. The uniformity and constancy of $G$ is transferred to the Quantum Pushing gravity through the uniformity in space the radiation pressure $\mathrm{p}_{\mathrm{o}}=\phi_{\mathrm{o}} \mathrm{E}_{\mathrm{o}} / \mathrm{c}$. Recalling that the ratio $\left(\sigma_{i} / m_{i}\right)=\mathrm{A}_{\mathrm{o}}$ is the same for all particles [as shown in par.7 by Equation (7.0.3)], we obtain the gravitational constant $\mathrm{G}$ in terms of the micro-quanta constants 


$$
G=\mathrm{p}_{\mathrm{o}} \mathrm{K}_{\mathrm{n}} \mathrm{A}_{\mathrm{o}}^{2} / \pi .
$$

This demonstrates that considering the "Gravitational" mass to be the "source" of Gravitational field is a misconception. Classical physics denounced more than a century ago that Inertial mass and Gravitational mass are numerically equal within 1 part on $10^{12}$. This proof would have been sufficient to abolish the concept of gravitational mass, but only today we can affirm that the "Gravitational" mass does not exists. Only the Inertial mass does exist and generates, through the mutual-shielding of the micro-quanta flux, the Quantum Pushing Gravity between particles. This generation of the Gravitational force gives full significance to the Strong version of the Equivalence principle, which is sometimes enounced in explicit form: "the generation of Gravitational force is due to the same physical agent (i.e. micro-quanta) that generates the Inertial forces.

The Newton's Gravitational pulling force has been, after all, the origin of other misconceptions, such as the black holes produced by the unlimited gravitational collapse, reducing stars to material points. Evidently an unphysical concept, which does not occurs when the gravitational collapse is not generated by "aristotelic" properties of the mass substance (which remains constant during the unlimited collapse), but depends on the pressure exerted by micro-quanta on the particles of a large celestial body. Since the great pressure $p_{o}$ is finite, the internal pressure of bodies cannot become infinite and the collapse ceases. This also happens when, during the collapse of a Supernova. the shrinking stops and comes out a neutron star. This class of misconceptions is not good for Physics. Many physicists don't accept the black holes. General Relativity theory, originally elaborated on a mathematical formalism that should avoid any misconception, actually it adopts in the practical formulations (such as the Schwarzschild integration) the "gravitational mass", which is signalled by the gravitational constant G. As a consequence, even G.R. predicts erroneously the formation of black holes.

\subsection{Gravitational force on the cosmological scale}

A notable result of the new Paradigm is the definition of the extended Quantum Pushing gravity. To take into account the rare collisions that micro-quanta do between them, a definition of the Pushing gravity force has been made (The New Cosmology, 2013) introducing in Equation (6.1) the exponential attenuation $\exp \left(-r / L_{o}\right)$ due to the micro-quanta that, mutually colliding within the volume of the missing beam joining two particles [which is normally devoid of micro-quanta travelling along it, as it can be assumed for distances $x<<L_{o}$ \}. This originates some scattered micro-quanta that travel just along the missing beam, which then reduces its capacity of generating the Quantum pushing gravity force between very far particles. So the correct value of Extended Pushing gravity is given by

$$
F_{g r}=\sigma \mathrm{p}_{\mathrm{o}} \mathrm{K}_{\mathrm{n}}\left(\sigma / 2 \pi r^{2}\right) \quad \exp \left(-r / L_{o}\right)
$$

where $L_{o}$ is the mean free path of micro-quanta crossing the universe. This equation becomes important at cosmological distances because $L_{o} \approx 1.31 \times 10^{26}$ equals the Hubble's length. As a consequence, the Static-evolutive universe, ruled by the Extended Pushing gravity, avoids by intrinsic nature the gravitational cosmic collapse that frightened the cosmologists in the first decades of past century. In the ' 20 Einstein proposed to introduce, in absence of known physical phenomena, a repulsive force based on an empirical constant to avoid the cosmic collapse of its static model.

The so called Cosmological constant, recently retrieved in the Accelerating model, has no clear physical meaning, since it has been assumed to dummy a reduction of Gravitation between galaxies. It is not an unknown repulsive force. If Einstein had known the exponential attenuation present in the Quantum Pushing gravity, he didn't hypothesise the cosmological constant.

Modern cosmologists and astronomers do not retrieve the obsolete Cosmological constant on the present cosmology. The authors of Accelerating universe affirmed to trust in the "guessing genius" of Einstein, but unfortunately they showed also not to believe (promoting the so called expansion of space) in the Law of Inertia born from the same mind.

\subsection{Cosmological Redshift of Photons Due to Collisions with Micro-Quanta}

The energy reduction of photons coming from far galaxies can be given in terms of the continuous collisions with the flux of (free) micro-quanta during photon travel. Being the photon constituted by an assembly of polarised micro-quanta softly linked between them (see par.7bis) at any collision with one (free) micro-quantum the photon leaves, on the average, one linked micro-quantum. Due to the thermodynamic nature of this process, the photon energy reduces exponentially at any mean free path $L_{o}=1.31 \times \times 10^{26}$ of micro-quanta. According to this physical process, the photon energy $\mathrm{hv}(x)$ shows a reduction $(\mathrm{d} v / \mathrm{v})$ at any path equal to the fraction $\left(\mathrm{d} x / L_{o}\right)$. 
In other words $\mathrm{d} v / v(x)=-\mathrm{d} x / L_{o}$ which gives

$$
v(x)=v_{\mathrm{o}} \exp \left(-x / L_{\mathrm{o}}\right) .
$$

Substituting in the definition of redshift $\mathrm{z}=\mathrm{v}_{\mathrm{o}} / v(x)-1$, one obtains

$$
\mathrm{z}=\exp \left(x / L_{\mathrm{o}}\right)-1
$$

which describes the redshift of photons travelling across the micro-quanta that collide on them during the time $t=$ $x / \mathrm{c}$. New Cosmology allows to calculate the distance $x$ of galaxies after measuring their redshift

$$
x=L_{\mathrm{o}} \ln (1+z) .
$$

This equation allows us to obtain the mean free path $L_{\mathrm{o}}$ calculating the distance $x_{\mathrm{o}}=L_{\mathrm{o}}$ of the sources whose measured redshift is $\mathrm{z}_{\mathrm{o}}=e-1=1.72 \ldots$

The tight formal correspondence between Micro-quanta paradigm and Plasma-redshift depends on the fact that both concepts consider two analogous thermodynamic interactions: a) collisions between photons and microquanta , b) collisions between photons and electron-plasma. This last interaction is probably more confined than the first, which is able to describe also the generation of strong inertial forces upon all neutral particles.

\section{Part 3. Advancement in Particle Dynamics at High Velocity (beyond Special Relativity).}

\section{The Physical Reality Underlying Special Relativity}

First of all, let's recall the fundamental role of the micro-quanta paradigm in giving new physical authority to Special relativity, whose weak dynamical structure was elaborated by Einstein (1905) using kinematical equations only. That unusual work aimed to demonstrate the general Law of Dynamics at high velocities, gave rise in the first decades of past century to enthusiasm among the mathematicians and disappointment between physicists. Notwithstanding the high popularity reached by the young Einstein, physicists felt difficulties to accept a dynamical theory built on kinematical tools. They made publicly the counter-elaboration of several paradoxes aimed to show the internal weakness of S.R. Some physicists guessed the existence of an underlying physical reality that might give physical substance to the relativistic dynamics. Unfortunately, this suggestion didn't obtain empirical demonstration by discovering some new physical reality.

Some doubts on S.R. continued when Arthur Compton in 1923 was awarded the Nobel Prize for the experiment showing the interaction of X-rays with the atomic electrons of graphite. This result did reinforce the Einstein's quanta of light, but at the same time revealed that the internal weakness of S.R. was in the absence of any dynamical equation (such as the Compton's one) in the incongruous demonstration of the Dynamical Law. To this subject one may read the recent paper "Suspending the Principle of Relativity" (Eskew, 2016).

In the subsequent decades the practical applications of relativistic equations were confirmed by experiments, particularly in the field of high energy particles. The elaboration of new paradoxes didn't cease, but physicists had argue that S.R. worked well at high velocities. Unfortunately this reinforced the wrong interpretations that attribute relativistic effects to the alleged physical properties of space and time, which are pure mathematical parameters organising the logical Scenario where the physical phenomena do happen. In particular the good capacity of G.R. metrics in explaining the astronomical observations (es. gravitational lens) reinforced the conviction that G.R. was conceived as Observational theory, because Einstein himself knew that G.R. (surely lacking of gravitational waves) wasn't a gravitational theory based on quanta. The famous dispute between Einstein and the physicists of the Physical Review about detection of GW, opened the run to the "gravitational antennas" that, after half a century of attempts, gave only discussed results. A new experimental result (C.Y. Lo, 2012) showed that, contrary to the easy generalization of G.R., the increment of thermal energy of metal bodies heated from 100 to $600{ }^{\circ} \mathrm{C}$ does not increase the weight, which in facts decreases. The phenomenon of the negative thermogravimetry has been confirmed more recently by a russian academic (Dmitriev, 2015).

How the micro-quanta flux generates the equations of Special Relativity is shown in detail in the paper "Physical Phenomena and Theoretical Problems" (Michelini, 2010). The demonstration is retrieved in APPENDIX 1.

The momentum of a particle moving with velocity $\mathbf{v}$ across the flux of micro-quanta was found to be

$$
\mathbf{q}_{I}=\left[\sigma_{l} \phi_{\mathrm{o}} \mathrm{E}_{\mathrm{o}} / f_{\mathrm{o}} \mathrm{c}^{2}\right] \mathbf{v} /\left(1-\mathrm{v}^{2} / \mathrm{c}^{2}\right)^{1 / 2}=\left[\sigma_{l} \phi_{\mathrm{o}} \mathrm{E}_{\mathrm{o}} / f_{\mathrm{o}} \mathrm{c}^{2}\right] \mathbf{v} / \beta
$$


where $\sigma_{i}$ is the particle cross section, $\phi_{\mathrm{o}}$ the flux of quanta, $\mathrm{E}_{\mathrm{o}}$ the energy and $f_{\mathrm{o}}$ the frequency of micro-quanta. There are no doubts: the structure of the momentum of particles is the relativistic one, when we define the term in brackets as thr rest mass $m_{i}$ of the particle having cross section $\sigma_{i}$

$$
m_{i}=\sigma_{i}\left[\phi_{\mathrm{o}} \mathrm{E}_{\mathrm{o}} / f_{\mathrm{o}} \mathrm{c}^{2}\right] .=\sigma_{i}\left[\mathrm{p}_{\mathrm{o}} / f_{\mathrm{o}} \mathrm{c}\right] .
$$

This definition of the rest mass of particles was unknown to the Special Relativity. The micro-quanta paradigm consents this information because we use a dynamical Law for calculating the total momentum released to the particle by the micro-quanta flux during a simultaneous collision of $\mathrm{N}_{\mathrm{i}}=\left[\sigma_{l} \phi_{\mathrm{o}} \tau_{\mathrm{o}}\right]$ micro-quanta, where $\tau_{\mathrm{o}}=1 / f_{\mathrm{o}}$ $=\lambda_{0} / \mathrm{c}$ is the time spent by a micro-quantum during recoiling under the repulsive force of the electric charge of particle. With the obvious substitutions, Equation (7.0) can be written in the canonical form

$$
\mathbf{q}_{i}=\left(\mathrm{m}_{i} / \beta\right) \mathbf{v}
$$

which contains two fundamental results.

The first is the correct (quantum-relativistic) structure of momentum calculated through collisions between microquanta and the particle with cross section $\sigma_{l}$ and velocity v. The operation giving Equation (7.0) consists in collecting and summing all $\Delta \mathrm{q}_{i}$ released by all micro-quanta incident on the particle. This generates and defines the relativistic mass $\left(\mathrm{m}_{i} / \beta\right)$ of particles which move across the flux of micro-quanta. Let's observe that this description recalls the Higgs' mechanism originating the rest-mass of particles defined by Equation (7.1). The notion of the micro-quanta flux strongly reduces the complex mechanism firstly devised by P. Higgs in 1964 . We will return on this subject in a next paper. There is no need of particular fantasy to imagine that when the $\gamma$-photons (constituted by a rotating pair electron-positron) under particular conditions collapses, the charges $\left(\mathrm{e}^{+}, \mathrm{e}^{-}\right)$disappear. The word coined to describe this phenomenon (annihilation) is unnecessarily dramatic. It is more correct to think that each charge, constituted of about $10^{21}$ micro-charges, loses the forces that compelled the micro-charges to remain tightly compacted, so the last ones move quickly to join the micro-charges of opposite sign. Due to the presence of some magnetic field, the two populations of micro-charges begin to rotate each other, determining the structure of two ordinary photons that substitute, in the equality of energy and momentum, the initial $\gamma$-.ray. This appears to be a viable representation of the Higgs' problem.

The second result is the definition of the inertial mass generated in proportion with the cross section. From Equation (7.0.1), the constant $\mathrm{A}_{\mathrm{o}}=\sigma_{i} / m_{i}$ can be put in the form

$$
\mathrm{A}_{\mathrm{o}}=\left[f_{\mathrm{o}} \mathrm{c} / \mathrm{p}_{\mathrm{o}}\right]
$$

where the value of $\mathrm{A}_{\circ}$ can be calculated from Equation (6.2)

$$
\mathrm{A}_{\mathrm{o}}=\left(\mathrm{G} / \mathrm{K}_{\mathrm{n}} p_{\mathrm{o}}\right)^{1 / 2}=5.13 \times 10^{-11}\left(\mathrm{~m}^{2} / \mathrm{kg}\right) \text {. }
$$

Recalling that the energy of any quanta is given by the product (Planck's constant $x$ frequency), the microquantum energy is defined $\mathrm{E}_{\mathrm{o}}=\mathrm{h}_{\mathrm{o}} f_{\mathrm{o}}$. Substituting the frequency $f_{\mathrm{o}}=\mathrm{E}_{\mathrm{o}} / \mathrm{h}_{\mathrm{o}}$ in the preceding equation we obtain the proper Planck's constant of micro-quanta

$$
\mathrm{h}_{\mathrm{o}}=\mathrm{c}^{2} / \mathrm{A}_{\mathrm{o}} \phi_{\mathrm{o}}
$$

that, by substitution gives $\mathrm{h}_{\mathrm{o}} \cong 1.30 \times 10^{-75}$, a value much less than standard Planck's constant.

This result gives the micro-quanta wavelength $\lambda_{0}=h_{o} c / E_{0} \cong 4.054 \times 10^{-35}$. All values of micro-quanta constants are third figure rounded to avoid exaggerated confidence since in their computation is present the gravitational constant. Also the classical Planck's length $l_{P}=\left(\mathrm{h} \mathrm{G} / \mathrm{c}^{3}\right)^{1 / 2} \cong 4.041 \times 10^{-35}$ contains the constant $\mathrm{G}$. A brief note on the accuracy of numerical gravitational values is reported at the end of Table 1.

The constants above reported open the door to the hypothesised micro-charges $\pm \Delta$ e that are ruled by the same law ruling the elementary charges

$$
\pm \mathrm{e}^{2} / \mathrm{h}= \pm(\Delta \mathrm{e})^{2} / \mathrm{h}_{\mathrm{o}}
$$

so we have $\Delta \mathrm{e}= \pm \mathrm{e}\left(\mathrm{h}_{\mathrm{o}} / \mathrm{h}\right)^{1 / 2} \cong 1.40 \times 10^{-21} \pm \mathrm{e}$.

The long sought possibility of describing the electric field in space through disseminated micro-charges, (as was cherished by P.W. Brigdman, Nobel prize 1946) appears now an objective that can be reached. 
Table 1. Principal constants of micro-quanta. This second normalization is linked to the quantisation of Newton's Gravitational force, which becomes the pulsating Quantum Pushing Gravity generated by collisions between particles and micro-quanta

\begin{tabular}{|c|c|c|}
\hline Constants of Micro-quanta & Numerical values & Comments \\
\hline - $\mathrm{E}_{\mathrm{o}}$ energy of micro-quantum & $9.62 \times 10^{-33}[$ Joule] & $\mathrm{E}_{\mathrm{o}}=\mathrm{Gm}_{\mathrm{n}}^{2} / \mathrm{x}_{\mathrm{o}}$ \\
\hline - $\phi_{\mathrm{o}}$ Flux of micro-quanta & $1.35 \times 10^{102}\left[\mathrm{~m}^{-2} \mathrm{~s}^{-1}\right]$ & Flux of free micro-quanta \\
\hline $\begin{array}{l}-\mathrm{x}_{\mathrm{o}}=\left(\mathrm{c} / \phi_{\mathrm{o}}\right)^{1 / 3} \text { average distance } \\
\text { between two micro-quanta }\end{array}$ & $6.06 \times 10^{-32}[\mathrm{~m}]$ & $\begin{array}{l}\text { The average density of micro-quanta in space is } \\
n=\phi_{\mathrm{o}} / \mathrm{c}\end{array}$ \\
\hline $\begin{array}{l}-\Delta \sigma=x_{0}^{2} \text { average area upon } \\
\text { which hits the missing beam }\end{array}$ & $3.67 \times 10^{-63}$ & $\begin{array}{l}\text { Constant fraction of cross section hit by one micro- } \\
\text { quantum }\end{array}$ \\
\hline $\begin{array}{l}\text { - } p_{o}=E_{o} \phi_{o} / c \text { energy density of } \\
\text { micro-quanta }\end{array}$ & $\begin{array}{l}4.33 \times 10^{61} \\
{\left[\text { Joule } / \mathrm{m}^{3}\right]}\end{array}$ & $\begin{array}{l}\text { Equals the radiation pressure of micro-quanta upon } \\
\text { particles }\end{array}$ \\
\hline $\begin{array}{l}\text { - } \mathrm{A}_{\mathrm{o}}=\sigma_{i} / m_{i} \text { constant ratio of any } \\
\text { particle }\end{array}$ & $5.13 \times 10^{-11}\left[\mathrm{~m}^{2} / \mathrm{kg}\right]$ & $\begin{array}{l}\text { Comparison between Newton's gravitation and } \\
\text { Quantum Pushing gravity. Value from eq (7.0.3). }\end{array}$ \\
\hline$-\sigma_{i}=\mathrm{A}_{\mathrm{o}} m_{i}$ & {$\left[\mathrm{~m}^{2}\right]$} & $\begin{array}{l}\text { The cross-section is proportional to the mass of } \\
\text { each particle. }\end{array}$ \\
\hline$-\sigma_{\mathrm{n}}$ nucleon cross-section & $8.57 \times 10^{-38}\left[\mathrm{~m}^{2}\right]$ & - \\
\hline$-\sigma_{\mathrm{e}}$ electron cross-section & $4.67 \times 10^{-41}\left[\mathrm{~m}^{2}\right]$ & - \\
\hline $\begin{array}{l}-\mathrm{h}_{\mathrm{o}}=\mathrm{c}^{2} / \mathrm{A}_{\mathrm{o}} \phi_{\mathrm{o}} \text { second Planck's } \\
\text { constant }\end{array}$ & $\begin{array}{l}1.30 \times 10^{-75}\left[\mathrm{~kg} \mathrm{~m}^{2} \mathrm{~s}^{-}\right. \\
\left.{ }^{1}\right]\end{array}$ & Obtained through micro-quanta constants \\
\hline $\begin{array}{l}-\lambda_{\mathrm{o}}=\mathrm{h}_{\mathrm{o}} \mathrm{c} / \mathrm{E}_{\mathrm{o}} \text { micro-quantum } \\
\text { wavelength }\end{array}$ & $4.054 \times 10^{-35}[\mathrm{~m}]$ & Obtained through micro-quanta constants \\
\hline$-\Delta \mathrm{e} / \mathrm{e}=\left(\mathrm{h}_{\mathrm{o}} / \mathrm{h}\right)^{1 / 2}$ micro-charge & $1.40 \times 10^{-21}$ & “ “ “ “ \\
\hline
\end{tabular}

NOTE on the numerical values of gravitational constants in Table 1.

The low-accuracy pervading gravitational results depends on the fact that $\mathrm{G}$ shows the greatest uncertainty between other fundamental interactions, whose uncertainty is less than $1 \mathrm{ppm}$. A Conference on the measurements of $\mathrm{G}$ (organised at London 1998, two centuries after the Cavendish experiment) showed that the best measurements made with methods - showed in the value of $\mathrm{G}$ an average difference around $1500 \mathrm{ppm}$, notwithstanding that specific errors of each measurement were 75 and $90 \mathrm{ppm}$. These results, that were confirmed in 2010 by the CODATA Committee, are in contrast with the tenet that gravity is a property of the masses.

The point of view of micro-quanta Paradigm on the observed discrepancies in the measurement of G is clear. Since the measured small gravitational forces a time average of small pulsating forces, there is (in principle) no surprise on the observed results. Besides, the definition of the Quantum Pushing force between two particles does not take into account that, for small times, a third particle may remain placed exactly along the missing beam joining the considered gravitational pair. This means that, for small times, the third particle does not give contribution to the measurement and the resulting $G$ is overestimated since in the Newton's equation the masses are generally determined by their weight obtained from balances, so even the third particle is considered. This brief note shows that Quantum Pushing gravity considers even the gravitational shield between masses, which was always denied by the classical physics.

\section{7.bis. Some Comments on the New Physical Realities}

Let's briefly comment the characteristics of the new physical objects (micro-quanta and micro-particles) whose constants are shown in Table 1. Micro-quanta are present in all fields of physics. For instance they participate to the modelling of the elementary particles, whose mass is originated by simultaneous collisions (E. M. interaction with the charge). If the flux $\phi_{0}$ is isotropic each incident quantum is balanced by an opposite incident quantum, so the (isolated) particle does not feel any force. Generally the simultaneous collisions determine pushing forces due to some missing beam originated by the mutual shielding between two particles. For instance any nucleon undergoes simultaneous collisions with $\mathrm{N}=\sigma_{n} \phi_{\mathrm{o}} \tau_{\mathrm{o}} \approx 1.5 \times 10^{22}$ micro-quanta. Any particle takes its stable characteristics (mass, spin, etc) just from the permanent simultaneous collisions with the flux $\phi_{\mathrm{o}}$. Some physical pushing forces (Gravitation, Strong and Weak forces, depending on distances between particles) are transmitted by some flux anisotropies, i.e. missing beams due to mutual shielding of two particles.

It may be interesting to sketch the role of the new micro-charges (micro-particles) which are primarily subject to the force from particle charges and to the impact of micro-quanta. Probably these micro-particles form rotating pairs with little masses which reproduce (in extremely reduced scale) some ordinary neutral objects. 
I think that micro-charges may explain some reactions between elementary particles where there is annihilation of charges or some reactions where two charges (electron and positron) suddenly appear from (apparently) nothing. I don't think that opposite charges annihilate. It's more likely that an elementary charge, under some very high field, releases its $\mathrm{N}_{\Delta_{\mathrm{e}}}=(\mathrm{e} / \Delta \mathrm{e})=7.17 \times 10^{20}$ micro-charges. When two opposite elementary charges (particle antiparticle) collide, both collapse so the mix of opposite micro-charges determines the formation of a pair of photons with $\mathrm{N}_{1}$ and $\mathrm{N}_{2}$ pair of micro-charges $\left(\Delta \mathrm{e}^{+}, \Delta \mathrm{e}^{-}\right)$so to conserve the energy and momentum. In other words, the stage of micro-charges is necessary to explain some (apparently) mysterious reactions between elementary particles.

Some researches project to detect the so called "Dark matter", which is an exotic way to indicate the search of a second Planck's constant. Some teams (Messina, 2014) intend to detect a new kind of particles that show gravitational effects, but do not emit E.M. radiation. Others are oriented on searching a new Planck's constant, for instance in Gravitational waves detection. To this subject I would recall that the recent "battage" of mass media on the discovery of the so called Einstein's GW does not show human respect toward Einstein. In fact he didn't accept that GW may be detected from cosmos. He simply affirmed that waves generating Gravitation are the same that generate Inertia. I minded this particular strong form of the Equivalence principle along my research. Since micro-quanta are the waves that generate Inertia of masses, then micro-quanta are the waves that generate Quantum Pushing gravity, i.e. the most accurate description of the Gravitational force.

An other possible interpretation of the role of micro-charges may be attributing them the mass that has been determined through Planck's satellite up to the limit of Near universe (Hubble's radius). Since the not specified "Obscure" energy may be neglected (let's recall that in the Static-evolutive universe the micro-quanta flux shows an enormous energy density that puts no physical problems since the micro-quanta do not practically collide among them, being their mean free path equal to about $10^{26} \mathrm{~m}$ ), the Planck's measurements (2014) show that the average density of matter in the universe is due $15,4 \%$ to baryonic mass (density $1.53 \times 10^{-27} \mathrm{~kg} / \mathrm{m}^{3}$ ) and $84.6 \%$ to Dark mass (density $8.37 \times 10^{-27} \mathrm{~kg} / \mathrm{m}^{3}$ ). Dark matter results 5.53 times more abundant than ordinary baryonic matter. This is about the same ratio found by Zwicky in 1930, attributing all the obscure mass to the dense (not luminous) dwarf stars representing the ultimate evolution of the luminous stars (Principal Sequence). Since the micro-charges cannot obviously generate photons, the density indicated by Planck satellite probably will contain some different types of obscure matter.

In the general confusion about theoretical demonstration of Dark Matter, it's worth signalling a research (Sigurdson, 2016) that intend to verify the correctness of the observations made by Zwicky (1930) naming obscure matter the great number of old or extinct stars.

\subsection{Micro-quanta are hidden in Newton's law of Inertia}

This paragraph wants to show the "Copernican" character of this work. Copernicus explained at beginning of 1600 the idea of the ancient greek philosopher Aristarcus, which sustained the heliocentric nature of solar system. Now we want to show that Newton's idea about the Inertia of bodies probably anticipated the present physical concept of micro-quanta more than 3 centuries ago.

The masterly Newton's law $\boldsymbol{F}=-m(\mathrm{~d} \mathbf{v} / \mathrm{dt})$ is in general presented in physics' manuals with the derivative notation. However this relation proves not to be accurate at the elementary particle scale, whose size is always finite. Newton showed scientific acumen specifying that his law of Inertia depends on some unknown properties of the Spatium absolutum, about which he humbly affirmed "Hypotheses non fingo". He was (correctly) convinced that the physical space differs from the mathematical space, which cannot introduce physical effects on bodies. Probably he suspected that the inertia of masses might depends on some physical agent, analogously to the "corpuscular light" he introduced to explain his experiments in optics. Dealing with Inertia, probably Newton thought of a flux of "corpuscular entities" with high constant velocity, which hit on moving particles producing a braking force when the particles accelerate and a pushing force when they decelerate. This phenomenon of Inertia remains also when the motion of bodies is studied within interplanetary space (high-level void) because micro-quanta fill all space. This fact induced the (incorrect) conviction that void space introduces physical effects on particles.

In current language, the "corpuscular entities" are named micro-quanta, i.e. very small quanta that can explain the Inertia of the elementary particles. The space where do happen inertial-gravitational phenomena, appears to be Euclidean to the astronomical observation, in contrast with the presence of gravitational fields that require (in relativistic language) a curved space. This means that inertial and gravitational phenomena are not due to different "properties" of space, but to the presence of a flux of micro-quanta which generates both phenomena.

In some sense Newton was the precursor of the physics of Micro-quanta, because he assumed a Law of inertia 


$$
\boldsymbol{F}=-m_{i}\left(\Delta \mathbf{v} / \Delta \mathrm{t}_{0}\right)
$$

where the acceleration is accurate respect to some unit of time supplied everywhere by an unknown physical reality, present in space. Notwithstanding it was unknown, Newton was able to pursue his work about the Dynamics of solar system, taking the fixed stars as reference frame of the planet positions. Of course, our physical reality has to satisfy this requirement even respect to the far masses of the universe.

May the ratio of finite increments appearing in the Law of inertia be assumed as ratio between two infinitesimal quantities? Mathematicians assume that the finite incremental ratio may be always substituted with derivative notation. Of course, the benefit of the Calculus of Variations in performing usual computations cannot be denied. But it's worth to remember that the analysis of physical phenomena requires to think of finite quantities. This is the logic of micro-quanta paradigm which requires that inertial forces encountered by an accelerating particle depend on the characteristics of the physical reality originating the inertia of bodies.

Let's consider a moving particle whose momentum $\mathbf{q}_{i}=\left(m_{i} / \beta\right) \mathbf{v}$ is given by Equation (7.0.2) derived from microquanta. Newton's Law of inertia deals with concepts of classical physics, so we consider the classical momentum of particles $\mathbf{q}_{i}=m_{i} \mathbf{v}$ which gives the inertial force through the finite increments of momentum divided by the finite increment of time

$$
\boldsymbol{F}=\Delta \mathbf{q} / \Delta \mathbf{t}=-m_{i} \Delta \mathbf{v} / \Delta \mathbf{t}
$$

where the time $\Delta \mathrm{t}$ is supplied by the average distance between adjacent micro-quanta $x_{0}=6.06 \times 10^{-32}$ given in Table 1. Two successive micro-quanta collide upon particle cross section with average time interval $\Delta \mathrm{t}_{0}=\mathrm{x}_{0} / \mathrm{c}=$ $2.02 \times 10^{-40}$, This is the correct time unit to measure the variation of velocity $\Delta \mathbf{v}$ originating the dynamical effects of the particle acceleration. Notice that the micro-quanta Paradigm doesn't offer systems of reference that can be hardly utilised in Dynamics (such as the inertial systems of S.R.). It offers much more: the time interval of the micro-quanta collisions upon particles. The variation of velocity $\Delta \mathbf{v}$ is inexorably revealed by this natural detector, originating a force of inertia that brakes the accelerating particles (or pushes the decelerating ones). In some sense any particle works as an accelerometer that records all deviations from the rectilinear uniform motion. How can these concepts be translated in mathematical equations?

A general procedure may be described in terms of the Operational Analysis in physics elaborated by Percy W. Brigdman. In his last years he published "Reflections of a physicist" (Brigdman, 1980) whose Preface contains a clear exposition :

"The new Analysis was primarily concerned with the new attitude toward physical concepts with which the physicist was meeting the crisis brought about by the discovery of new facts in the domain of relativity and quantum phenomena. The essence of the attitude is that the meanings of one's terms have to be found by an analysis of the operations which one performs in applying the term in concrete situations, or in verifying the truth of statements, or in finding the answer to questions".

Let's consider an accelerated particle moving through an uniform flux of quanta, with velocity increment $\Delta \mathbf{v}$ within the unspecified time $\Delta$ t. Let's put $\mathrm{E}_{\mathrm{o}}$ the energy of quanta and $\sigma_{i}$ the particle cross section. Experience has been accumulated in collisions of photons upon particles, releasing some momentum and energy. This is the case of the Doppler shift that calculates the photon frequency $f$ when colliding with the receiver. The classic scheme of calculating $f$ assumes that photons with frequency $f_{\mathrm{o}}$ travel across a medium with proper velocity (as it happens for acoustical waves moving through the air, producing the Doppler effect of sirens) reaching the receiver (for instance a particle) with frequency

$$
f=f_{\mathrm{o}}(\mathrm{c}+\mathrm{v}) /(\mathrm{c} \pm \mathrm{vs})
$$

where $\mathrm{v}$ is the velocity of receiver (i.e. particle) and $\pm \mathrm{v}_{\mathrm{S}}$ is the receding (approaching) velocity of the source of photons. This equation is used for astronomical purposes where the signal reaches the receiver from one side only. It is also compatible with the relativistic Doppler. It appears clear that this scheme is inadequate to describe the balance of momentum released by micro-quanta on both sides of the particle, described with the cross section $\sigma_{i}$ normal to the velocity (not the whole spherical surface $4 \sigma_{i}$ offered to the isotropic flux). The classical scheme is inadequate to describe the micro-quanta collisions for two reasons: 1) the particle receives simultaneously pairs of opposite quanta incident forward and behind, 2) micro-quanta exist from indefinite time, have no source and their energy $\mathrm{E}_{\mathrm{o}}$ does not change when approaching the particle. The cause is due to the fact that the source of sound originates acoustic waves in the surrounding medium, whereas the micro-quanta are single waves (much smaller than photons) that behave as small projectiles in the void space. 
To be short, Newton's Law of inertia is due to the variation of the collision rates on both sides of moving particles, due to the variation of velocity $\Delta \mathbf{v}$ revealed through a physical flux of quanta that (on the average) collide regularly i.e. at constant interval. Let's consider an accelerated particle which receives forward an increased collision rate $r_{\mathrm{f}}$ $=\sigma_{l}\left(\phi_{\mathrm{o}} / 2\right)(\mathrm{c}+\Delta \mathrm{v}) / \mathrm{c}$ and behind a reduced collision rate $r_{\mathrm{b}}=\sigma_{l}\left(\phi_{\mathrm{o}} / 2\right)(\mathrm{c}-\Delta \mathrm{v}) / \mathrm{c}$. Since each recoiling micro-quantum releases the small momentum $2 \mathrm{E}_{0} / \mathrm{c}$, it follows that the net inertial force opposing the motion is

$$
-\boldsymbol{F}=2\left(\mathrm{E}_{\mathrm{o}} / \mathrm{c}\right)\left(r_{\mathrm{f}}-r_{\mathrm{b}}\right)=\left(\mathrm{E}_{\mathrm{o}} / \mathrm{c}\right) \sigma_{i} \phi_{\mathrm{o}}(\Delta \mathbf{v} / \mathrm{c}) .
$$

Substituting $\sigma_{i}=\mathrm{Ao}_{\mathrm{o}} m_{i}$ and putting $\mathrm{E}_{\mathrm{o}} \phi_{\mathrm{o}} / \mathrm{c}=\mathrm{p}_{\mathrm{o}}$, one gets

$$
-\boldsymbol{F}=\mathrm{p}_{\mathrm{o}} \mathrm{A}_{\mathrm{o}} m_{i} \Delta \mathbf{v} / \mathbf{c} .
$$

Since the term $\mathrm{p}_{\mathrm{o}} \mathrm{A}_{\mathrm{o}} / \mathrm{c}$ (recalling eq.7.3) can be written $\mathrm{p}_{\mathrm{o}} \mathrm{A}_{\mathrm{o}} / \mathrm{c}=f_{\mathrm{o}}=\mathrm{c} / \mathrm{x}_{\mathrm{o}}=1 / \Delta \mathrm{t}_{\mathrm{o}}$, substituting in Equation (7.1.4) we obtain the Newton's Law of Inertia $\boldsymbol{F}=-m_{i} \Delta \mathbf{v} / \Delta \mathrm{t}_{\mathrm{o}}$ where it is specified the small time

$$
\Delta \mathrm{t}_{\mathrm{o}}=\mathrm{x}_{\mathrm{o}} / \mathrm{c}=2.02 \times 10^{-40}
$$

that acts as the unit of time between two successive collisions of micro-quanta.

Notice that this equation may be useful to analyse in great detail some very rapid phenomena that presently are not understandable.

In other words, nature provides a formidable device denouncing inertial forces that brake accelerating particles or push decelerating particles. This explains also the physical reason of the principle of Inertia: "any body free from external forces remains indefinitely in its state of uniform motion".

Whatever be placed in the universe, the motion of an accelerated body denounces an inertial force which is opposite to any external force acting on the motion of the body.

Contrary to the great difficulties experienced in half century of detecting from Cosmos the so called Gravitational waves, the best way of detecting the existence of micro-quanta is given by the accelerometers widely used on vehicles, both spacecraft and terrestrial, to record any variation occurring in the velocity. Particularly useful are the so called Black boxes installed on board that give important details on the causes that produced shocks on vehicles, everywhere they are placed. It appears evident that in the whole space there is a physical reality (whose presence is not signalled by E.M. waves) which can be easily signalled by its capacity to produce on free bodies some inertial forces that can be recorded.

The energy density of micro-quanta is due to the high concentration in space (equal to $\phi_{o} / \mathrm{c}=4.5 \times 10^{93} \mathrm{quanta} / \mathrm{m}^{3}$ ) multiplied by the micro-quantum energy $E_{0}=9.62 \times 10^{-33}$, which gives the energy density $p_{o}=E_{o} \phi_{o} / c=4.33 \times 10^{61}$ Joule $/ \mathrm{m}^{3}$. This quantity may appear perilously high. On the contrary, it's a quiet reality because micro-quanta $d o$ not locally collide each other. These quanta are so small that their mean free path equals the Hubble's distance $L_{\mathrm{o}}$ $\approx 1.31 \times 10^{26}$, which rules the redshift of photons. Why this well known distance coincides with the mean free path of micro-quanta? Obviously the suggested relation must be stringent. The explanation is that the continuous collisions of micro-quanta upon photons reduce gradually their energy hv as well experienced by astronomical observations. The mechanism is simple: each free micro-quantum scatters one of the $n=\mathrm{hv} / \mathrm{E}_{\mathrm{o}}$ low energy polarised micro-charges constituting the photon, according to the thermodynamic rule

$$
\mathrm{d}(\mathrm{h} v) / \mathrm{h} v=\mathrm{d} v / v=-\mathrm{d} x / L_{\mathrm{o}}
$$

which shows by integration (see par. 6.3) the genesis of the new cosmological redshift that links the distance $x$ travelled by photons to the "damage" produced on their structure by collisions with micro-quanta

$$
(\mathrm{z}+1)=\exp \left(x / L_{\mathrm{o}}\right) .
$$

The flux $\phi_{0}$ of micro-quanta is uniform throughout the universe? The flux appears to be linked to the large masses of the universe, thus explaining the accuracy of the inertial systems offered by the fixed stars (or masses of far galaxies). The linkage to, the large masses shows a mean free path of micro-quanta several times less that the characteristic size of masses. For instance, in the Sun the m.f.p. is equal to R/65. Reduced effects are predicted on planets. For instance Jupiter shows a m.f.p. equal to R / 4.8.

We showed that in the world of micro-quanta is possible studying phenomena between particles that involve time intervals as little as $\Delta \mathrm{t}_{\mathbf{0}} \approx 10^{-40}$. If we want to explain why the acceleration of a body produces the inertial force, 
we are compelled to find its origin on the same flux of micro-quanta that has yet proved to explain the Gravitation through the Quantum Pushing gravity force (par. 6).

Probably the first proof of the existence of micro-quanta was given, without knowing it, by Newton himself, whose thought shows to day a new freshness. Correspondently, the renounce to a physical interaction generating the inertial forces, weakened the Einstein's thought about the linkage between Inertial and Gravitational interactions.He was notably involved in the problem of the aberration of light, which connects the Quantum Gravitational phenomena and the astronomical observations..

The kinematical structure of Special relativity was unable to find the origin of the inertial mass because microquanta flux and cross section of particles didn't exist when S.R. appeared. This is the reason why the inertial forces on the moving particles are attributed by S.R. to pseudo-properties of void-space. This argument regards also General relativity whose metrics takes into account all aberrations originated by transmission of gravitational phenomena with light signals. This property is very useful to astronomers in deciphering some strange observations (for instance the gravitational lens).

\section{Part 4. Inadequacy of the Classical Theory of Star Formation}

\section{The Discovery of the Bok's Globules and the Impasse of Classical Physics}

In 1945 the astronomer Barth Bok discovered some opaque gas globules in some regions of the Milky Way, showed masses somewhat greater than star masses which gave new impulse to the Jeans' theory published in the first ' 900 . For instance the observations of some gas globules with radius $\mathrm{R}$ between $8 \times 10^{14}$ and $5 \times 10^{15}$ showed that their masses were about $2 \mathrm{M} \odot$ and $50 \mathrm{M} \odot$. The mass figures attributed by astronomers to the Bok's globules are near the values predicted by the Jeans' critical mass undergoing instability

$$
\mathrm{M}_{\mathrm{J}}=(\pi / 6) \mathrm{c}_{\mathrm{s}}{ }^{3} /\left(\mathrm{G}^{3} \delta\right)^{1 / 2}
$$

which depends on the density $\delta$ of galactic gas (assumed around $10^{-22} \div 10^{-21}$ ) in different regions of Milky Way. This classical theory didn't give results due to Jeans' hypothesis that the globule contraction happens in free fall. This is manifestly wrong because the velocity of contraction would be comparable with dense meteorites falling in atmosphere, where they fire and evaporate. The contraction of large gas globules (size around $10^{15}$ ) is similar to the gravitational accretion of galactic gas upon small inert bodies placed in the middle of Galaxies

Besides, the high densities (around $10^{-16} \div 10^{-14}$ ) that astronomers attributed to the observed globules show a level of contraction around $10^{6}$ times the initial gas density. This requires that globules began contracting when the Jeans' initial radius $R_{0}$ was considerably higher (about $10^{17}$ ) than the observed radius. This reinforce the objection that classical theory is not able to explain how the globules may attain the ignition temperature of the nuclear fusion. On the contrary, astronomers believe that Bok's globules show the characteristics of star incubators. In fact it appears strange that present level of science and technology is not yet able to explain the formation of stars, whose birth is known from centuries through the observation of Novae within Milky Way, whose present rate of discovery equals about $10-11$ per year.

Classical physics believed that all energy heating large globules (up to $10^{3} \mathrm{M} \odot$ ) was given by gravitational contraction. This tenet was supported by the old experience with adiabatic compression of gases in mechanical devices. Heating gases up to high temperatures implies the generation of intense radiation by rapid compression. If the radiation remains in a pressure chamber perfectly insulated from energy leakage, gas remains at constant temperature (ideal radiation cavity). In space the radiation escapes easily from celestial bodies with low density, such as the young Bok's globules. Newton's pulling gravity (a classical force, not supported by quanta) is unable to give up energy directly to particles during contraction, since it transmit principally momentum between particles. On the contrary, Quantum Pushing gravity is able to transmit energy due to its quantum pulsating structure, implying Compton's equation to release energy from any single quantum, This happens for photons as well as for other quanta.

New data appear necessary to explain the formation of stars, since classical physics did not show if gravitational energy is sufficient to heat the cold Bok's globules up to the ignition temperature $\mathrm{T}_{\mathrm{ig}}=10^{7} \mathrm{~K}$ of nuclear $\mathrm{p}$-p fusion. In fact the classical energy balance doesn't guarantee that the radius $r_{x}$ is small enough to reach in the core the temperature $\mathrm{T}_{\mathrm{ig}}$. This uncertainty depends on the fact that the atom collision rate of falling gas may give rise to a gas pressure that overcomes the dynamic pressure originating from the falling mass of gas. Jeans suggested that high thermal power is generated in free falling collapse. But the phenomenon of falling meteorites, that fire by impact on atmosphere, does not apply because even meteorites cannot reach free fall in atmosphere. Any falling mass within an atmosphere shows a final limiting velocity. 


$$
\mathrm{G} \mathrm{M}^{2} / r_{x}=\mathrm{C}_{H} \mathrm{M} \Delta \mathrm{T}_{\mathrm{x}}=\mathrm{C}_{H} \mathrm{M}\left(\mathrm{T}_{\mathrm{ig}} / \zeta\right)
$$

Let's consider what happens in the classical balance of power during the globule contraction

$$
-\left[\mathrm{G} \mathrm{M}^{2}(\mathrm{dR} / \mathrm{dt}) / R^{2}\right]\left[1-\exp \left(\mathrm{K}_{r} R / l\right)\right]=\mathrm{C}_{H} \mathrm{M}(\mathrm{dT} / \mathrm{dt})
$$

where $(\mathrm{dR} / \mathrm{dt})$ is the contraction velocity, while the exponential attenuation takes into account the absorbed radiation, linked to the photon mean free path $l=m_{p} / \delta(r) \sigma_{\mathrm{f}}$ and to the fraction $K_{r}=\mathrm{h} \Delta \mathrm{v} / \mathrm{hv}=\mathrm{hv} / \mathrm{m}_{\mathrm{e}} \mathrm{c}^{2}$ of photon energy leaved in Compton's collisions with electrons.

Let's notice that Equation (8.1) does not allow to describe the globule contraction. In fact eliminating the time in both sides and integrating on $\mathrm{dR}$ and $\mathrm{dT}$, we obtain an equation linking the globule radius $\mathrm{R}$ in function of the average temperature increment $\Delta \mathrm{T}$, without any possibility to calculate the incubation time necessary to reach the ignition temperature of the $\mathrm{p}-\mathrm{p}$ nuclear fusion.

Putting the initial radius $\mathrm{R}_{\mathrm{o}}$ (which appears to be much larger than the radius $\mathrm{R}_{\mathrm{ob}}$ of the observed Bok's globules) we obtain

$$
\left(\mathrm{GM} / \mathrm{R}-\mathrm{GM} / \mathrm{R}_{\mathrm{o}}\right)\left[1-\exp \left(\mathrm{K}_{r} R / l\right)\right]=\mathrm{C}_{H} \Delta \mathrm{T}
$$

where $\Delta \mathrm{T}$ is the average globule temperature. The term with $\mathrm{R}_{\mathrm{o}}$ is negligible. Since the globule density grows during time, the escaping radiation reduces during contraction. This happens when the radius $\mathrm{R} \approx 10^{15}$. We may begin to count the contraction starting from $\mathrm{R} \approx 10^{15}$. To reach the lowest fusion temperature in nature (the $\mathrm{p}-\mathrm{p}$ reaction of Hydrogen shows an ignition temperature of $10^{7} \mathrm{~K}$ ) the globule average temperature has to reach $\Delta \mathrm{T}_{\mathrm{x}}$ $\approx 10^{7} / \zeta$, where the parameter $\zeta$ is about $14 \div 21$. From Equation (8.2) one gets the ratio $M_{x} / R_{x}$ when the globules reach the ignition in the interior

$$
\mathrm{M}_{\mathrm{x}} / \mathrm{R}_{\mathrm{x}} \approx\left(\mathrm{C}_{\mathrm{H}} / \mathrm{G}\right) \Delta \mathrm{T}_{\mathrm{x}}=6 \times 10^{13}\left(10^{7} / \zeta\right) \approx 3.6 \times 10^{19} .
$$

The ratio $M_{x} / R_{x}$ of globules is conceptually different from the analogous ratio of stars $\left(M^{*} / R^{*}\right)$ deduced from the study of the Initial mass function of Stars (Kroupa P. 2002) coming from the observation in astronomy and astrophysics. Conversely, the ratio given by Equation (8.3) can be only calculated, because when a globule reaches the p-p ignition temperature (about $10^{7} \mathrm{~K}$ in Hydrogen gas), no signal can be observed since the incipient radiation of nuclear fusion cannot (for some time) escape from the large mass of globules. This is the reason why the ratio $\mathrm{M}_{\mathrm{x}} / \mathrm{R}_{\mathrm{x}}=3.6 \times 10^{19}$ shows a unique value for all Novae.

Actually the presence of about $25 \% \mathrm{He}$ rises the ignition up to $1.8 \times 10^{7} \mathrm{~K}$. This limit increases further when the p$\mathrm{p}$ cycle is substituted by the $\mathrm{CNO}$ cycle which requires greater ignition temperatures. The following Table 2 gives the observed ratios $\left(\mathrm{M}^{*} / \mathrm{R}^{*}\right)$ for different types of stars constituting the population of the Main Sequence. Mass is expressed in $\mathrm{M} \odot$ and radius in $\mathrm{R} \odot$. In the last column SI units are indicated. This Table shows that classical physics in some cases works well.

From Equation (8.3) one may obtain the radius $R_{x}$ of the globule when, for instance, we consider a typical mass $M_{x}=1.58 \times 10^{31}$, i.e. the mass of the globule that originated the Sun. Then from Equation (8.3) it follows

$$
\mathrm{R}_{\mathrm{x}}=1.58 \times 10^{31} / 3.6 \times 10^{19}=4.4 \times 10^{11} .
$$

\begin{tabular}{|c|c|c|c|}
\hline Mass $\left(\mathrm{M}^{*} / \mathrm{M} \odot\right)$ & Radius $\left(\mathrm{R}^{*} / \mathrm{R} \odot\right)$ & $\left(\mathrm{M} * / \mathrm{R}^{*}\right)$ & $\left(\mathrm{M}^{*} / \mathrm{R}^{*}\right) \quad(\mathrm{kg} / \mathrm{m})$ \\
\hline 40 (giant) & 18 & 2.22 & $6.28 \times 10^{21}$ \\
\hline $18(")$ & 7.4 & 2.43 & $6.51 ،$ \\
\hline 1.10 (medium) & 1.05 & 1.05 & $3.0 “$ \\
\hline $1.00 \mathrm{Sun}$ & 1.00 & 1.00 & 2.83 “ \\
\hline 0.93 (medium) & 0.93 & 1.00 & 2.8 “ \\
\hline 0.69 ( small $)$ & 0.74 & 0.93 & $2.6 “$ \\
\hline $0.21(“)$ & 0.32 & 0.65 & $1.8 “$ \\
\hline $0.10(“)$ & 0.13 & 0.77 & 2.2 “ \\
\hline
\end{tabular}

All information we may obtain from classical physics is that the time duration of the gravitational collapse initiates when $\mathrm{R} \approx 10^{15}$ and terminates when $\mathrm{R} \approx 4.4 \times 10^{11}$.

Table 2. Ratios $\left(\mathrm{M}^{*} / \mathrm{R}^{*}\right)$ for different types of stars constituting the population of the Main Sequence are reported in function of the observed parameters. [M.V. Zombeck, 1990. Handbook of Space Astronomy and Astrophysics] 
In conclusion, the present attempt of describing the correct evolution of the gravitational collapse shows that classical physics is inadequate to solve the dilemma. The problem does not come from pure mathematical difficulties, but from some inadequacy in the classical model of globule "collapse". This is proved in the following paragraph where the time of star incubation is faced, through Boltzmann's theory of Transport in gases, involving the phenomenon of gravitational accretion.

\section{Formation of Stars by Gravitational Accretion of Gas on Inert Masses}

There is another possibility of studying the globule collapse dynamics. The progressive contraction of gas globules (surely much slower than Jeans' free fall hypothesis which neglected the obstacle due to the increasing density of the accreted gas) may be described by the gravitational accretion of galactic gas upon inert masses $\mathrm{M}_{\mathrm{o}}$. The gravitational accretion of galactic gas upon a dead star - with very little residual radiation - can increase notably its mass (M. Michelini, 2007) giving origin to some obscure Supermasses (up to 3.7 million times the Sun mass) discovered in the last decades in the middle of Milky Way (Schoedel et al., 2002).

The gravitational accretion model appears more accurate to the problem of gas globule contraction with increasing temperature studied a century ago by J. Jeans assuming the concept of gravitational instability that showed high inaccuracy in calculating the velocity of contraction. At present level of understanding, the observed Bok's globules follows the scenario of mass accretion upon some gravitational "seeds" constituted by fragments of Supernovae explosions, planetary nebulae condensed, concentrations of cosmic powder, etc. This choice is enforced by considering the high densities (about $10^{-16} \div 10^{-14}$ ) that astronomers attributed to the observed globules showing a contraction equal to about $10^{6}$ times the initial galactic gas density. This fact requires that globules began to contract when the Jeans' initial radius $\mathrm{R}_{\mathrm{o}}$ was considerably higher (about 100 times) than the observed radii $\mathrm{R}_{\mathrm{ob}}$ between $10^{15} \div 5 \times 10^{15}$. In other words the observed Bok's globules cannot be the initial stage imagined by Jeans.

Let's now assume $M_{o}$ to be the mass of inert bodies (intermediate between the mass of Jupiter and Uranus) placed in high gas density regions of Galaxies, which begin accreting mass from the gas, mostly hydrogen. Let's consider a gas globule, whose escaping radiation is centred in the far infrared since their surface temperature does not exceed a few degrees Kelvin (making them obscure by contrast) when the observed globule radius is around $\mathrm{R}$ $\approx 10^{15}$, so to seems incubator of future stars. This fact has been discovered by observing the Bok's globules which appear as obscure bodies against a luminous background of galactic powder. Putting $\mathrm{M}(r)$ the accreting mass, the accretion rate is calculated through the velocity and density of gas that cross its boundary (surface $4 \pi r^{2}$ ) constituted by a thin shell of galactic gas

$$
\mathrm{dM}(r) / \mathrm{dt}=4 \pi r^{2} \mathrm{u}(r) \delta(r)
$$

where the radial velocity $\mathrm{u}(r)=\tau\left[\mathrm{GM}(t) / r^{2}\right]$ is given by the gravitational acceleration of the mass $\mathrm{M}(r)$ multiplied by the average time of flight $\tau(r)=l(r) / \mathrm{v}_{\mathrm{g}}(r)$, where $l(r)=\mathrm{m}_{\mathrm{g}} / \sigma_{\mathrm{g}} \delta(r)$ is the mean free path of atoms entering the mass $\mathrm{M}(\mathrm{t})$ of gas, $\mathrm{m}_{\mathrm{g}}$ is the mass of Hydrogen and $\sigma_{\mathrm{g}}=8.8 \times 10^{-21}$ its cross section. Finally the atom velocity $\mathrm{v}_{\mathrm{g}}(r)=\left[2 \mathrm{kT}_{\mathrm{o}}(r) / \mathrm{m}_{\mathrm{g}}\right]^{1 / 2}$ depends on the increasing gas temperature $\mathrm{T}_{\mathrm{o}}(r)$ at boundary produced by the gravitational accretion. Substituting these parameters in Equation (9.1) one obtains the accreting rate on the gravitational mass $\mathrm{M}(\mathrm{t})$

$$
\mathrm{dM}(r) / \mathrm{dt}=4 \pi \mathrm{GM}(r) \mathrm{m}_{\mathrm{g}} / \sigma_{\mathrm{g}}\left[2 \mathrm{kT}_{\mathrm{o}}(r) / \mathrm{m}_{\mathrm{g}}\right]^{1 / 2} .
$$

The gravitational accretion increases the mass $\mathrm{M}(r)$ from $\mathrm{M}_{\mathrm{o}}$ up to the mass $\mathrm{M}_{\mathrm{x}}$ achieved when the temperature in the core reaches the ignition $\left(10^{7} \mathrm{~K}\right)$ of the nuclear $\mathrm{p}-\mathrm{p}$ fusion. The accretion rate depends on the ratio $\mathrm{M}(r) /$ $\mathrm{T}_{\mathrm{o}}(r)^{1 / 2}$ which is minimum when the accretion is at beginning and increases when the mass $\mathrm{M}(r)$ reaches the maximum $\mathrm{M}_{\mathrm{x}}$ in correspondence with the ignition of the nuclear fusion. After this turning point, about $70 \div 96 \%$ of the mass is rapidly blown off by the strong radiation of the nuclear fusion. Separating the variables in Equation (9.2) one obtains

$$
\mathrm{dM} / \mathrm{M}(r)=4 \pi \mathrm{G}\left(1.48 \times 10^{-8} / \sqrt{\operatorname{To}(r)}\right) \mathrm{dt} .
$$

Integrating both sides, the star incubation time results

$$
\mathrm{t}^{*}=8.07 \times 10^{16} \sqrt{[\mathrm{To}]} \ln \left(\mathrm{M}_{\mathrm{x}} / \mathrm{M}_{\mathrm{o}}\right)
$$

where we have to specify the average boundary temperature [To] during the accretion from the initial inert mass $M_{o}$ up to the final mass $M_{x}$ As to the inert mass, we may refer to the surface temperature of isolated inert masses. An example is given by the external solar planets. Deducing the contribution of the Sun's radiation, their 
temperatures range from about $40^{\circ} \mathrm{K}$ (Pluto) up to about $43^{\circ} \mathrm{K}$ (Neptune). On the other hand, the surface temperature of the accreted mass $\mathrm{M}_{\mathrm{x}}$ just before the ignition of nuclear fusion, continues to be cold (less than $100^{\circ} \mathrm{K}$ ) because the gravitational heating is distributed on a large mass.

For instance let's verify the radiation emission $P_{x}$ from a typical mass $M_{x}=8 M \odot \quad$ calculated by averaging between two estimated mass $(2 \mathrm{M} \odot$ and $50 \mathrm{M} \odot$ ) of the observed Bok's globules

$$
\mathrm{P}_{\mathrm{x}}=4 \pi \mathrm{R}_{\mathrm{x}}{ }^{2} \mathrm{~K}_{\mathrm{S}} \mathrm{T}_{\mathrm{ox}}{ }^{4} \text { (watt) }
$$

where the radius $\mathrm{R}_{\mathrm{x}} \approx \mathrm{M}_{\mathrm{x}} / 3.6 \times 10^{19} \approx 4.4 \times 10^{11}$ is obtained from Equation (8.3) which balances the gravitational power and the heating power released to the mass

$$
\mathrm{M}_{\mathrm{x}} / \mathrm{R}_{\mathrm{x}} \approx\left(\mathrm{C}_{\mathrm{H}} / \mathrm{G}\right) \Delta \mathrm{T}_{\mathrm{x}} \approx 3.6 \times 10^{19} .
$$

The final radiation power $P_{x}$ is confined on the IR spectrum, like the emissions of the Bok's globules observed by the astronomers that estimated values of the order of $10^{24}-10^{25}$ watt. The final radiation $\mathrm{P}_{\mathrm{x}}$ is of the same order, so the surface temperature $\mathrm{T}_{\mathrm{ox}}$ (in eq.9.5) results comprised between $56^{\circ} \mathrm{K}$ and $100^{\circ} \mathrm{K}$ according to the low or high estimate of $\mathrm{P}_{\mathrm{x}}$. To resume, the boundary temperature during the incubation appears to increase from about $40^{\circ} \mathrm{K}$ up to about $95^{\circ} \mathrm{K}$. Since the radiation in the past was much lower than the final radiation power $\mathrm{P}_{\mathrm{x}}$, due to the small ratio of surfaces $\left(r^{2} / \mathrm{R}_{\mathrm{x}}^{2}\right)$, the average surface temperature [ $\left.\mathrm{T}_{\mathrm{o}}\right]$ must be a little shifted towards the final value. Being the dependence of the star incubation time on the surface temperature not dramatic, we assume simply $\left.\left[\mathrm{T}_{\mathrm{o}}\right]=(40+95)+3=69^{\circ} \mathrm{K}\right)$.

Substituting in Equation (9.4) the following star incubation time $t^{*}$ can be found assuming a mean globule mass $\mathrm{M}_{\mathrm{x}}=1.2 \mathrm{M} \odot=2.4 \times 10^{30}$ :

$$
\begin{array}{lll}
\text { - initial mass } \mathrm{M}_{\mathrm{o}}=10^{26} & \mathrm{t}^{*}=8.2 \times 10^{18} \mathrm{sec} & (210 \mathrm{Gyr}) \\
\text { - initial mass } \mathrm{M}_{\mathrm{o}}=10^{27} & \mathrm{t}^{*}=5.16 \times 10^{18} \mathrm{sec} & (163 \mathrm{Gyr}) \\
\text { - initial mass } \mathrm{M}_{\mathrm{o}}=10^{28} & \mathrm{t}^{*}=3.64 \times 10^{18} \mathrm{sec} & (115 \mathrm{Gyr}) \\
\text { - initial mass } \mathrm{M}_{\mathrm{o}}=10^{29} & \mathrm{t}^{*}=2.11 \times 10^{18} \mathrm{sec} & (67 \mathrm{Gyr}) \\
\text { - initial mass } \mathrm{M}_{\mathrm{o}}=6 \times 10^{29} & \mathrm{t}^{*}=0.92 \times 10^{18} \mathrm{sec} & (29 \mathrm{Gyr}) .
\end{array}
$$

Results show the dependence of the incubation time on the initial mass. In substance, these values show clearly that the time needed for accreting globules and consequently igniting the p-p nuclear fusion, goes from a few Gyr up to hundreds of Gyr.

It is useful to verify the hypothesis adopted (i.e. the free fall motion) in classical Jeans' theory of collapsing globules. In Gravitational accretion, the average growth velocity (assuming an inert mass $\mathrm{M}_{0}=10^{26}$ corresponding to an incubation time $\mathrm{t}^{*}=8.2 \times 10^{18}$ ) from the initial value $r_{\mathrm{o}}$ up to the maximum $\mathrm{R}_{\mathrm{x}} \approx 4.4 \times 10^{11}$, results $[\mathrm{v}]=\mathrm{R}_{\mathrm{x}} / \mathrm{t}^{*}$ $=0.53 \times 10^{-7} \mathrm{~m} / \mathrm{s}$, that is much lower than the free fall velocity $\left(10^{3}-10^{5} \mathrm{~m} / \mathrm{s}\right)$ hypothesised by Jeans.

The slow accretion velocity is due to the tortuous path that each gas molecule (due to its angular momentum that would keep it indefinitely orbiting in absence of collisions) follow for long time before reaching the accreting mass. The theory of the gravitational "collapse" ignored the accreting process of the globules.

\section{9.bis. An Analysis of the Structure of the Milky Way Galaxy.}

The introduction of the star Incubation time through the Gravitational accretion model makes possible to establish a galactic balance between the luminous stars, the "extinct" (low luminous) star and the mass of gas involved in the Star forming model.

It is experimentally ascertained that in the Milky Way there are about $10^{11}$ luminous stars of various masses, with total mass estimated around $10^{11} \mathrm{M} \odot$.

Astronomical observations (Gnedin et al., 2010) showed recently a gravitational mass of $6.9 \times 10^{11} \mathrm{M} \odot \quad$. Some years ago, Planck's Satellite measured an invisible/obscure mass equal to 5.7 times the luminous mass, that is $5.7 / 6.7=85.1 \%$ of the total mass. Consequently the mass of luminous stars (up to now never specifically measured) results

$$
6.9 \times 10^{11} \mathrm{M} \odot \quad \times 14.9 \%=1.028 \times 10^{11} \mathrm{M} \odot \quad .
$$

The discrepancy between the measuremen6ts is not pronounced. The luminous stars of the Main Sequence increase

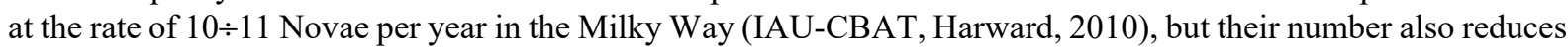
due to the gradual Hydrogen consumption, that transforms bright stars in low-luminosity dwarf-stars of various 
colour index. The surface temperature is high, but their radius gradually reduces, so these stars remain generally invisible. In any case, many of them have been observed and studied. Those affirming that these stars do not exists, because the Big bang Age of universe is too little to be comprehensive of them, are simply wrong.

The process of Star Formation gradually transform the galactic gas in luminous stars through the Gravitational accretion upon some inert masses (seeds) that are present in galaxies, for instance through the production of fragments from the explosion of Supernovae. But also the formation of planetary systems may become (when the dominant star will cease, diffusing its gaseous mass in the neighbourhood) good "seeds" for the gravitational accretion of gas. In par. 9 it has been shown that the time of incubation of Novae depends on the size of the initial mass. Some of the mature globules (just reaching the ignition temperature of $\mathrm{H}$ nuclear fusion) will produce a Nova within some dozen of Giga-years, whereas other will require hundreds of Giga-years. It is clear that the evolution of a bright galaxy depends on the average incubation time of Novae.

What we know is that about 10,5 Novae per year are born from our galactic furnace. The count of Novae is done from the XVII century, but in the ' 60 the rate were a few Novae per year. However with the advent of orbiting telescopes, the rate of Novae has rapidly increased up to 10 per year, showing that this count is approaching the real value (independent from technologies). How many "channels" of Novae are contemporaneously present in the galaxy? This depends on the quantity of gas present in the Active regions of galaxy and on the number of "seeds"(inert masses) at disposal. Let's compare the Formation of Novae and other galactic objects in the balance of the galaxy total mass

$$
6.9 \times 10^{11} \mathrm{M} \odot=1.028 \times 10^{11} \mathrm{M} \odot+\left[\mathrm{M}_{\mathrm{GA}}+\mathrm{M}_{\text {old }}+\mathrm{M}_{\mathrm{gas}}\right]
$$

where $M_{\text {old }}$ is the mass of the old (invisible) stars, $M_{\text {gas }}$ is the remaining mass of free gas and $M_{G A}$ is the mass under active Gravitational accretion that now produce yearly 10.5 Novae, whose average mass equals $1 \mathrm{M} \odot$, if calculated over an adequate time interval, The brackets in the preceding equation include the terms constituting the invisible/obscure mass, that equals $85.1 \%$ of total. Since the mass of each Nova does not change macroscopically during ageing (excepting Supernovae), the total number of stars is equal to the total number of Novae generated up to the present time $t_{0}$, taking the time origin at the birth of the first Nova

$$
6.9 \times 10^{11}=\int_{o}^{t o} f(t) d t
$$

where $f(t)$ is the rate of Novae, which is declining due to the continuous reduction of the mass of gas at disposal. Let's assume the exponential reduction $f(\mathrm{t}) \approx f_{\mathrm{o}} \exp \left(-\mathrm{t} / \mathrm{t}_{\mathrm{o}}\right)$, which takes the present rate of Novae (10.5 per year) observed on the Milky Way. So one gets $f(\mathrm{t}) \approx 28.6 \exp \left(-\mathrm{t} / \mathrm{t}_{\mathrm{o}}\right)$.

Substituting in Equation (A2.3) we finally obtain

$$
6.9 \times 10^{11}=28.6 \mathrm{t}_{\mathrm{o}}[1-\exp (-1)]=18.1 \mathrm{t}_{\mathrm{o}}
$$

so the present time $t_{o}$ equals $3.81 \times 10^{10}$ years after the first star. In practice the age of our structured galaxy amount to $38.1 \mathrm{Gyr}$, to which it must be added the incubation time of the first star.

\section{Part 4. The Growth of Matter Temperature in the Universe}

\section{Release of Energy Through Micro-Quanta Collisions on Particles}

The paradigm shows that micro-quanta generate Quantum gravitational Pushing force that actually pushes on particles with different effect respect to classical gravitational force. In fact Quantum gravitation pushes on particles through single micro-quanta incident at average time interval $\Delta \mathrm{t}_{\mathrm{o}}=\mathrm{x}_{\mathrm{o}} / \mathrm{c}$ where $\mathrm{x}_{\mathrm{o}}$ is the mean distance between two adjacent micro-quanta. This distance may be calculated from the flux $\phi_{0}$ since the average microquanta density in space is

$$
\mathrm{n}_{\mathrm{o}}=\left(\phi_{\mathrm{o}} / \mathrm{c}\right)=1 / \mathrm{x}_{\mathrm{o}}^{3}
$$

Recalling from Table 1 that $\phi_{\mathrm{o}}=1.35 \times 10^{102}$, one calculate $\mathrm{x}_{\mathrm{o}}=6.06 \times 10^{-32}$. Notwithstanding the small value of $\Delta \mathrm{t}$ $=\mathrm{x}_{\mathrm{o}} / \mathrm{c}=2.02 \times 10^{-40}$, any collision is distinct from the other. This ultrafine sequence of single collisions releases to the particle some quantum energy given by the Compton's equation

$$
\Delta \mathrm{E}=\left(\mathrm{E}_{\mathrm{o}}^{2} / m_{e} \mathrm{c}^{2}\right) .
$$

However this does not happens to all incident quanta, but only to quanta transmitting forces, such as the Pushing Gravitation, upon particles. In fact on the isolated particles (i.e. hit by unperturbed isotropic flux) each quantum 
does not transmit net force because the momentum it leaves is balanced by the opposite momentum leaved by a diametrically opposite incident quantum. This perfect statistical equilibrium is due to the high rate of micro-quanta collision on particles. For instance upon a nucleon are colliding $\sigma_{n} \phi_{0} \approx 6 \times 10^{64}$ quanta/second.

The Quantum Pushing gravity force $\boldsymbol{F}=(2 \mathrm{E} / \mathrm{c}) \psi(r)$ between two facing particles (cross section $\sigma)$ at distance $r$, is due to the recoiling beam of micro-quanta $\psi(r)=\Delta \sigma \phi_{0}\left(\Delta \sigma / 2 \pi r^{2}\right)$ colliding on a very small cross section $\Delta \sigma$ diametrically opposite to an equal $\Delta \sigma$ on which the beam $\psi(r)$, along the line joining the two particles, is absent due to the mutual shielding between particles (see par. 6). This asymmetry generates a net pushing force on each particle

$$
\boldsymbol{F}_{g r}=\left(2 \mathrm{E}_{\mathrm{o}} / \mathrm{c}\right) \Delta \sigma \phi_{\mathrm{o}}\left(\Delta \sigma / 2 \pi r^{2}\right) .
$$

Through this mechanism, any quantum of the missing beam $\psi(r)$ between two particles, releases to the electron the energy given by Equation (10.1) at intervals $\Delta \mathrm{t}_{\mathrm{o}}$, so on the average the particle receives a "continuous" power given by

$$
p_{\mathrm{e}}=\left(\mathrm{E}_{\mathrm{o}}^{2} / m_{e} \mathrm{c}^{2}\right) \psi(r)=\left(\mathrm{E}_{\mathrm{o}}^{2} / m_{e} \mathrm{c}^{2}\right)\left(\Delta \sigma \phi_{\mathrm{o}}\right)\left(\Delta \sigma / 2 \pi r^{2}\right)
$$

Calculating the value of $\Delta \sigma$ requires, by definition, that the rate of quanta collisions $\left(\Delta \sigma \phi_{\mathrm{O}}\right)$ be equal to $1 / \Delta \mathrm{t}_{\mathrm{o}}=$ $c / \mathrm{x}_{\mathrm{o}}$ which gives $\Delta \sigma=c / \phi_{\mathrm{o}} \mathrm{x}_{\mathrm{o}}$. Recalling Equation (10.1) and substituting $c / \phi_{\mathrm{o}}=\mathrm{x}_{\mathrm{o}}{ }^{3}$, one gets

$$
\Delta \sigma=\mathrm{x}_{\mathrm{o}}^{2}=\left(c / \phi_{\mathrm{o}}\right)^{2 / 3}=3.67 \times 10^{-63}
$$

which, substituted in Equation (10.3), gives the power generated by micro-quanta upon an electron that undergoes the Pushing gravity force from a nucleon at distance $r$

$$
\mathrm{p}_{e}=\left(\mathrm{E}_{\mathrm{o}}^{2} / m_{e} \mathrm{c}^{2}\right)\left(c / \mathrm{x}_{\mathrm{o}}\right)\left(\mathrm{x}_{\mathrm{o}}^{2} / 2 \pi r^{2}\right)
$$

This equation shows that $\mathrm{p}_{e}$ depends on the density of matter around the electron. Considering that almost all mass of matter is due to nucleons, we calculate the contribute of a body with radius $\mathrm{R}$ and density $\delta(r)$ to the gravitational power

$$
\mathrm{p}_{e}=5.61 \times 10^{-11} \mathrm{x}_{\mathrm{o}}^{2} \int_{0}^{R} 2\left[\delta(r) / m_{n}\right] \mathrm{d} r=1.23 \times 10^{-46} \int_{0}^{R} 2 \delta(r) \mathrm{d} r
$$

where $m_{n}$ is the mass of nucleons. At first approximation the term $\int_{0}^{R} 2 \delta(r) \mathrm{d} r$ equals $\left(\mathrm{M} / \mathrm{R}^{2}\right)$, so one obtains

$$
\mathrm{p}_{e} \approx 1.23 \times 10^{-46}\left(\mathrm{M} / \mathrm{R}^{2}\right) \text { watt }
$$

It is worth of noticing that the above result is obtained from well experienced principles of physics. They cannot be confused with hypotheses.

Let's consider the case of the Bok's globules present in the Milky Way which are believed to be incubators of stars. Bok's globules show masses so large to guarantee (after the blow off of the excess maa) both the birth of large star as well as of small stars. For instance, direct observations, as reported by M. Szpir (Sci. Am. 2001), showed $\mathrm{M} \approx 2$ solar masses as well as $\mathrm{M} \approx 50$. The mass of globules is always in excess respect to the mass of the imminent Nova.

What happens to the increment of the electron energy? The most simple answer may be just a little increase of the electron kinetic energy, which in the Hydrogen atom amounts to

$$
\mathscr{E}=1 / 2 \mathrm{~m}_{e} \mathrm{v}^{2}=1 / 2 \mathrm{~m}_{e} \alpha^{2} \mathrm{c}^{2}
$$

This hypothesis corresponds to an increment of the fine structure constant amounting to (d $\alpha / \mathrm{dt}) / \alpha=\mathrm{p}_{e} / \mathrm{m}_{e} \alpha^{2} \mathrm{c}^{2}$, which for $\mathrm{H}$ atoms within Bok's globules is of the order of $(\mathrm{d} \alpha / \mathrm{dt}) / \alpha \approx 2.8 \times 10^{-20} \mathrm{sec}^{-1}$.

$$
\mathrm{d} E / \mathrm{dt}=\mathrm{m}_{e} \alpha \mathrm{c}^{2} \mathrm{~d} \alpha / \mathrm{dt}=\mathrm{p}_{e} \approx 1.23 \times 10^{-46}\left(M / R^{2}\right)
$$


Such situation corresponds to the hypothesis of the increasing velocities of particles advanced in the past by the cosmologists of the Quasi-Steady state model. On the contrary, it is known that the Big bang model predicts a cooling of the universe due to its expansion.

However it is most probable that the excess of electron energy generates photon emission, in particular in the Hydrogen IR spectral series (Balmer, Paschen, Brakett, Pfund) which heats the surrounding mass, so the power $p_{e}$ has to be added to the gravitational power in the balance of the accreting mass in the Star Forming model.

\section{References}

Bridgman, P. W. (1980). Reflection of a physicist (retr. 1980). Arno Press.

Brigdman, P. W. (1952). La logica della fisica moderna (trad. V. Somenzi).

Brynjolfsson A. (2006). Magnitude-Rsdshift relation for SNe 1a, Time dilation and Plasma Redshift. arXiv: astro$\mathrm{ph} / 0602500 \mathrm{v} 1$.

Brynjolfsson, A. (2005). Redshift of photons penetrating a hot plasma. arXiv: astroph/0401420v3,2005.

Crothers, S. (2016). A critical analysis of LIGO's recent detection of $G W$ caused by merging Black holes. Hadronic Journal, 39, 2016.

Eskew, R. C. (2016). Suspending the Principle of Relativity. Applied Physics Research, 8(2), 82.

Gnedin, O. Y., Brown, W. R., Geller, M. J., \& Kenyon, S. J. (2010). The Mass Profile of the Galaxy to 80 kpc. The Astrophysical Journal Letters, 720(1), L108.

IAU-CBAT. (2010). List of Novae in the Milky Way, 2010. Harward Univ.

Kroupa P. (2002). The initial mass function of Stars. Science, 295(5553), 82-91.

Li, Z. (2015). Physics Essay: Six Baseless and Irrational Problems in Modern Cosmology. Applied Physics Research, 7(6), 56.

Lo C. Y. (2012). On the Weight reduction of Metals due to the Temperature increment. Glob. Jour. Science Frontier Research, 2012.

Lopez-Corredoira, M. (2003). Observational Cosmology: caveats and open questions in the standard model. arXiv preprint astro-ph/0310214.

Messina J. F. (2012). Question of Planck's constant in Dark Matter direct detection. APS.

Messina, J. F. (2014). Question on planckian Action in gravitational waves detection. APS.

Michelini, M. (2010). Physical Phenomena and Theoretical Problems Explained by the Micro-Quanta Paradigm. Applied Physics Research, 2(2), 90.

Michelini, M. (2011). The Gravitational power originating the Earthquakes and the Dansgaard-Oeschger catastrophic Events. Applied Physics Research, 3(1), 76.

Michelini, M. (2012). Beyond the Special and General Theories of Relativity. Appl. Phys. Res., 4.

Michelini, M. (2013). The New Cosmology Arising from the Quantum Pushing Gravity Interaction-The Case of Accelerating Universe. Applied Physics Research, 5(5), 67.

MNRAS. (2016). Monthly Notices R.A.S. March, 2016.

Rossler, O. E. (2016). A trap posed by Nature to Humankind. Univ. of Tubingen,

Schödel, R., Ott, T., Genzel, R., Hofmann, R., Lehnert, M., Eckart, A., ... \& Hartung, M. (2002). A star in a 15.2year orbit around the supermassive black hole at the centre of the Milky Way. Nature, 419(6908), 694-696.

Sigurdson, K. (2016). A speed Test for Dark Matter. Retrieved from www.physics.aps.org

Szpir, M. (2001). Scientific American, 2001.

\section{APPENDIX}

"Physical Phenomena and Theoretical Problems explained by the Micro-quanta Paradigm retriv. Applied Physics Research (M. Michelini Nov. 2010)

Let's consider a free particle moving through the flux of micro-quanta. The particle velocity is defined through Doppler as the effective velocity which changes the frequencies of the micro-quanta incident along the direction of motion. Namely, the frequency of quanta colliding in front is $v_{\mathrm{f}}=v_{\mathrm{o}}(1+\mathrm{v} / \mathrm{c})^{1 / 2} /(1-\mathrm{v} / \mathrm{c})^{1 / 2}$, whereas 
$v_{b}=v_{o}(1-v / c)^{1 / 2} /(1+v / c)^{1 / 2}$ is the frequency of quanta colliding behind. So, the effective velocity $v$ can be expressed, operationally, through the ratio $\left(v_{\mathrm{f}} / v_{\mathrm{o}}\right)=\left(v_{\mathrm{o}} / v_{\mathrm{b}}\right)$ between the frequency in front and the natural frequency $v_{0}$ of micro-quanta

$$
\mathrm{v} / \mathrm{c}=\left[\left(v_{\mathrm{f}} / \mathrm{v}_{\mathrm{o}}\right)^{2}-1\right] /\left[\left(\mathrm{v}_{\mathrm{f}} / \mathrm{v}_{\mathrm{o}}\right)^{2}+1\right] .
$$

For instance, a particle is at rest $(\mathbf{v}=0)$ respect to the incident isotropic flux when the frequencies in front and behind equal $v_{o}$.

Being the Doppler frequencies an intrinsic characteristic of the particle motion, the velocity $\mathbf{v}$ appears to be the effective or absolute velocity (i.e. not-depending on the reference systems) of the particle. Of course the frequency $v_{\mathrm{f}}\left(\right.$ or $v_{\mathrm{b}}$ ) might be hardly observed. But we may know the energy and momentum of the particle (eq.3) which gives its velocity on physical grounds.

This explains the accuracy of the solar system dynamics when referred to the "inertial" frame linked to large masses of the universe, because they are globally at rest respect to the local flux of micro-quanta.

The undulatory characteristics of the electromagnetic waves, as well as other waves, are defined by proportionality between energy and frequency. For usual electromagnetic waves the proportionality is given by the Planck's constant. For the very small energy $E_{0}=h_{0} v_{0}=h_{0} c / \lambda_{0}$ of micro-quanta the proportionality constant is much smaller. As later shown, we assume that the quantum wavelength $\lambda_{\mathrm{o}}$ equals the Planck's length $l_{\mathrm{P}}=\left(G h / \mathrm{c}^{3}\right)^{1 / 2} \cong 4.05 \times 10^{-35}$ which depends both on gravitational and inertial phenomena. This choice is congruent with the definition of the second Planck's constant as shown in Table 1 of the micro-quanta parameters.

Very important is the concept of simultaneous collisions of micro-quanta upon the particle cross section $\sigma_{i}$. The time a micro-quantum requires to bounce is of the order of $\tau_{\mathrm{o}}=2 \lambda_{\mathrm{o}} / c$, so the simultaneous collisions in front of the particle are $\mathrm{N}_{\mathrm{f}}=\sigma_{i} \phi_{\mathrm{f}} \tau_{\mathrm{f}}$, whereas behind are $\mathrm{N}_{\mathrm{b}}=\sigma_{i} \phi_{\mathrm{b}} \tau_{\mathrm{b}}$, where $\phi_{\mathrm{b}}$ and $\phi_{\mathrm{f}}$ are the perturbed fluxes behind and in front of the particle. To give an idea, the simultaneous collisions (duration time $\tau_{\mathrm{o}}$ ) of micro-quanta upon a nucleon are of the order of $10^{50}$, a fact which justifies the collision process as a stationary event. This originates the stable particles, as well as the short-lived particles due to the little $\tau_{\mathrm{o}}=2 \lambda_{\mathrm{o}} / \mathrm{c}=2.67 \times 10^{-43}$.

Whatever the numerical value of $h_{0}$, the momentum of a free particle moving within the quantum flux is given by the momentum received from backward collisions minus the momentum of forward collisions

$$
|\mathbf{q}|=N_{\mathrm{b}} E_{\mathrm{b}} / c-N_{\mathrm{f}} E_{\mathrm{f}} / c=\left(h_{\mathrm{o}} / \mathrm{c}\right) \sigma_{i}\left(v_{\mathrm{b}} \phi_{\mathrm{b}} \tau_{\mathrm{b}}-v_{\mathrm{f}} \phi_{\mathrm{f}} \tau_{\mathrm{f}}\right)
$$

which, recalling that $v_{j} \tau_{j}=1$, becomes

$$
\mathrm{q}=\left(E_{\mathrm{o}} / c v_{\mathrm{o}}\right) \sigma_{i}\left(\phi_{\mathrm{b}}-\phi_{\mathrm{f}}\right) .
$$

Free particles moving within the isotropic flux $\phi_{\mathrm{o}}$ maintain indefinitely their motion (principle of Inertia) since the micro-quanta do not create obstacles to stationary straight motions. The conservation of the unperturbed energy density $\ni_{0}=\phi_{0} E_{0} / c$ of micro-quanta requires that, during the collisions, the energy density in front and behind the particle, remains unchanged, although the related frequencies change with Doppler

$$
\ni_{\mathrm{o}} / 2=\phi=v_{\mathrm{b}} \phi_{\mathrm{b}} h_{\mathrm{o}} / \mathrm{c}=v_{\mathrm{f}} \phi_{\mathrm{f}} h_{\mathrm{o}} / \mathrm{c} .
$$

Substituting in this equation the Doppler frequencies $v_{\mathrm{f}}$ and $v_{\mathrm{b}}$, the resulting perturbed fluxes can be substituted in Equation (1a) obtaining the momentum of a free particle moving within the flux

$$
\mathbf{q}_{i}=\left[\sigma_{i} \phi_{\mathrm{o}} \tau_{\mathrm{o}} E_{\mathrm{o}} / c^{2}\right] \mathbf{v} /\left(1-\mathrm{v}^{2} / c^{2}\right)^{1 / 2} .
$$

This expression coincides with the relativistic momentum when the product in brackets defines the inertial restmass of particles originated by collisions with the micro-quanta flux

$$
\mathrm{m}_{\mathrm{o} i}=\left(\sigma_{i} \phi_{\mathrm{o}} \tau_{\mathrm{o}}\right) E_{\mathrm{o}} / c^{2}
$$

It defines also the proportionality between cross-section and mass of particles $\sigma_{i}=\mathrm{m}_{\mathrm{o} i} \mathrm{~A}_{\mathrm{o}}$ where $\mathrm{A}_{\mathrm{o}}$ is a constant for all particles

$$
\mathrm{A}_{\mathrm{o}}=c^{2} / \phi_{\mathrm{o}} \tau_{\mathrm{o}} E_{\mathrm{o}} .
$$

Let's remark that Equation (4) shows specifically that the rest-mass of a particle

$$
m_{\mathrm{o} i} c^{2}=\left(\sigma_{i} \phi_{\mathrm{o}} \tau_{\mathrm{o}}\right) E_{\mathrm{o}}=\mathrm{N}_{i} E_{\mathrm{o}}
$$

is equivalent to the energy of $\mathrm{N}_{i}=\left(\sigma_{i} \phi_{\mathrm{o}} \tau_{\mathrm{o}}\right)$ micro-quanta hitting simultaneously the particle. Notice that $\mathrm{N}_{i}$ is the inverse of the Compton ratio $\mathrm{K}_{\mathrm{o} i}=E_{\mathrm{o}} / \mathrm{m}_{\mathrm{o} i} c^{2}$. 
It shows specifically why there is equivalence between rest-mass and energy, as obtained in principle by Special relativity.

As we know, particles may disrupt under high energy collisions. This explains, for instance, that a particle of high energy $E_{\text {tot }}=\mathrm{N}_{i} E_{\mathrm{o}} /\left(1-\mathrm{v}^{2} / c^{2}\right)^{1 / 2}$ may disappear through a violent collision, releasing $\mathrm{N}_{i}$ micro-quanta which rearrange into different particles with the conservation of charge and energy.

This equation describes the inertial model of particles as persistent aggregates of micro-quanta. It shows specifically why there is equivalence between rest-mass and energy, as obtained in principle by Special relativity.

\section{Copyrights}

Copyright for this article is retained by the author(s), with first publication rights granted to the journal.

This is an open-access article distributed under the terms and conditions of the Creative Commons Attribution license (http://creativecommons.org/licenses/by/4.0/). 